\title{
ISOLATED INVARIANT SETS FOR FLOWS ON VECTOR BUNDLES
}

\author{
BY \\ JAMES F. SELGRADE
}

\begin{abstract}
This paper studies isolated invariant sets for linear flows on the projective bundle associated to a vector bundle, e.g., the projective tangent flow to a smooth flow on a manifold. It is shown that such invariant sets meet each fiber, roughly in a disjoint union of linear subspaces. Isolated invariant sets which are intersections of attractors and repellers (Morse sets) are discussed. We show that, over a connected chain recurrent set in the base space, a Morse filtration gives a splitting of the projective bundle into a direct sum of invariant subbundles. To each factor in this splitting there corresponds an interval of real numbers (disjoint from those for other factors) which measures the exponential rate of growth of the orbits in that factor. We use these results to see that, over a connected chain recurrent set, the zero section of the vector bundle is isolated if and only if the flow is hyperbolic. From this, it follows that if no equation in the hull of a linear, almost periodic differential equation has a nontrivial bounded solution then the solution space of each equation has a hyperbolic splitting.
\end{abstract}

1. Introduction. In analyzing solutions to an autonomous system of differential equations, a useful technique is to study the linearized equations. The globalization of this approach is studying a flow $f$ on a manifold by analyzing its tangent flow $T f$ on the tangent bundle to the manifold. (" $T$ " denotes the tangent functor-here applied to the map $f_{t}$ for each $t \in R$.) Our analysis concentrates on the isolated invariant sets of the flow which $T f$ induces on the projective bundle associated to the tangent bundle (each fiber is the projective space obtained from the tangent space). Certain isolated invariant sets are central to the study of $f$, for example, those corresponding to the tangent spaces to the stable and unstable manifolds of a hyperbolic critical point of $f$ are a repeller and an attractor, respectively, of the projective tangent flow.

While our investigation has been motivated by the desire to study flows on smooth manifolds in terms of isolated invariant sets of the projective tangent flow,

Received by the editors October 10, 1973 and, in revised form, January 28, 1974.

AMS (MOS) subject classifications (1970). Primary 34C35; Secondary 34A30, 54H20, 55F25, 58F15.

Key words and phrases. Isolated invariant set, vector bundle, associated projective bundle, linear flow, cross ratio, expansion point, isolating block, attractor, repeller, chain recurrence, Morse set, Morse decomposition, hyperbolic flow, almost periodic system. 
we have not proved any theorems specific to tangent flows. Our results do include several basic theorems relating the linear aspects of vector bundle flows to the notion of isolated invariant sets.

We consider a flow $F$ on a vector bundle over a compact metric space which, for each $t \in R$, is a vector bundle morphism (takes fibers to fibers, linearly). A flow on the base space lying under $F$ is denoted by $f$; and the pair $(F, f)$ is called a linear flow. Because of linearity, $F$ induces a flow $P F$ on the projective bundle associated with the vector bundle. The compactness of the projective bundle allows the use of techniques not available for the vector bundle.

$\S \S 2,3$ and 4 develop the necessary background and introduce some concepts useful in proving the main result treated in $\S \S 5,6$ and 7 . This result asserts that an isolated invariant set of $P F$ intersects each fiber in a disjoint union of linear subspaces together with closed arcs of 1-dimensional linear subspaces (Theorem 7.1). Also, in each fiber, there are only a finite number of distinct subspaces which can be traces of isolated invariant sets on that fiber (Theorem 7.4).

$\$ 8$ contains a brief summary of some recent work of Charles Conley on chain recurrence and Morse sets [6]. A Morse set means the intersection of an attractor and a repeller; so Morse sets are isolated. To each filtration of a flow there corresponds an ordered collection of Morse sets, and any collection arising in this way is called a Morse decomposition of the flow. The existence of filtrations of a flow depends on a weak recurrence relation defined in $\S 8$. A theorem in [6] states that a flow is "chain recurrent" if and only if there are no nontrivial filtrations.

In $\S 9$, we prove that a Morse set of $P F$ meets each fiber in one linear subspace (without exception). Furthermore, if the base is connected and the flow $f$ is chain recurrent on it, then each Morse decomposition of $P F$ corresponds to a splitting of the projective bundle into a direct sum of invariant subbundles. (An example shows that chain recurrence in the base is necessary.) In $\S 10$, we use these results to see that, if $f$ is chain recurrent on a connected base, then the zero section of the vector bundle is an isolated invariant set of $F$ if and only if $F$ is hyperbolic. Also, to each Morse set in a Morse decomposition over a chain recurrent, connected base, there corresponds an interval of real numbers which measures the exponential rate of growth of the orbits in that set. The intervals corresponding to different Morse sets are disjoint. This implies that there is a unique finest Morse decomposition and that $P F$ restricted to each of the Morse sets in this decomposition is chain recurrent. If the base space is a rest point or a periodic orbit, the growth rate intervals corresponding to Morse sets in the finest decomposition are points. This is not generally the case.

As a corollary, we prove a strengthened version of a result due to Robert Sacker and George Sell [18] concerning almost periodic differential equations. 
Their result states that if no equation in the hull of the almost periodic system, $\dot{x}=A(t) x$, has a nontrivial bounded solution, then the solution space of each equation in the hull splits into a direct sum of a subspace with exponential growth and a subspace with exponential decay. Our theorem on finest Morse decompositions over a chain recurrent base generalizes and refines this statement; in particular, the chain recurrence of $P F$ restricted to each Morse set is a novel feature.

The results presented here are taken from the author's doctoral thesis written under the direction of Charles Conley. The author wishes to thank Professor Conley for his invaluable assistance.

2. Flows on vector bundles and projective bundles. Let $(M, d)$ be a compact metric space. Let $(E, M, \pi, M)$ denote a vector bundle over $M$ as defined in [1]. $E$ is the total space, $\pi$ is the projection from $E$ onto $M$, and $M$ is the maximal atlas of charts. Charts, subbundles, quotient bundles, Whitney sums, and Riemannian metrics have their usual meaning [1], [13]. Unless otherwise stated, we will assume all vector bundle structures are continuous; and we will denote a bundle by its total space.

REMARK. Let $E$ be an $n+1$-dimensional bundle over $M$. For $b \in M$, let $R_{b}^{n+1}$ denote the fiber over $b$, i.e., $R_{b}^{n+1} \equiv \pi^{-1}(b)$. Then $R_{b}^{n+1}$ has a unique vector space structure.

Proposition 2.1. If $E^{\prime}$ is a closed subset of $E$ and $E^{\prime}$ intersects each fiber in a $k$-dimensional subspace $(1 \leqslant k \leqslant n+1)$, then $E^{\prime}$ is a $k$-dimensional subbundle of $E$.

Consider the subspace $E-Z$ of $E$ where $Z$ is the zero section of the vector bundle $E$. On $E-Z$ perform the identification corresponding to the following equivalence relation: For $b \in M$ and $v_{b}, w_{b} \in R_{b}^{n+1}-0, v_{b} \sim w_{b}$ if and only if there is an $\alpha \neq 0 \in R$ so that $v_{b}=\alpha w_{b}$. The equivalence class of $v_{b}$ will be denoted $\left[v_{b}\right]$. Let $P E$ denote the identification space with the quotient topology and $P \pi$, the induced projection from $P E$ onto $M$. With this topology, $(P E, M, P \pi)$ becomes a fiber bundle with fiber $P^{n}$, real $n$-dimensional projective space, and is called the projective bundle associated with $(E, M, \pi, M)$. Because of the linearity of the charts for $E$, they induce charts for $P E$ which will be referred to as linear bundle charts for $P E$.

Definition. For $b \in M$, let $P_{b}^{n}$ denote the fiber of $P E$ over $b$. For $0 \leqslant k \leqslant n$, a $k$-dimensional linear subspace of $P_{b}^{n}$ is the image under identification of a $k+1$-dimensional subspace of $R_{b}^{n+1}$.

DEfinition. A subset $H$ of $P E$ is a $k$-dimensional subbundle of $P E$ if its pull-back to $E$ by the identification map is a $k+1$-dimensional subbundle of $E$. 
DEFInItIon. $P E$ is said to be the direct sum of its subbundles $\left\{P E_{1}, \cdots, P E_{k}\right\}$ if $E$ is the Whitney sum of $\left\{E_{1}, \cdots, E_{k}\right\}$.

A flow on $M$ will be denoted by $f: M \times R \rightarrow M$ and sometimes by $\left\{f_{t}\right\}$, $f_{t}: M \rightarrow M$ for each $t \in R$. A flow on the total space $E$ will be denoted by $F$ or $\left\{F_{t}\right\}$. If $Y$ is a subset of $M$ (or of $E$ ) and $J$ is a subset of $R$, then $Y \cdot J \equiv f(Y \times J)$ (respectively, $Y \cdot J \equiv F(Y \times J)$ ). $(F, f)$ is a linear flow on the bundle $E$ if:

(1) $F$ and $f$ are flows on $E$ and $M$, respectively,

(2) $f_{t} \circ \pi=\pi \circ F_{t}$ for all $t \in R$, and

(3) $F_{t}: R_{b}^{n+1} \rightarrow R_{f_{t}(b)}^{n+1}$ is a linear isomorphism for each $b \in M$.

Because of the linearity, $F$ induces a flow on the total space $P E$ defined by $P F\left(\left(\left[v_{b}\right], t\right)\right) \equiv\left[F\left(\left(v_{b}, t\right)\right)\right]$ for all $b \in M,\left[v_{b}\right] \in P E$, and $t \in R$. The advantage in studying $P F$ instead of $F$ is that $P F$ is a flow on a compact metric space which lends itself to the study of isolated invariant sets [2], [7], [15], [26]. The canonical example of a linear flow on a vector bundle is a $C^{r}$-flow $(r \geqslant 1)$ on a compact manifold and its tangent flow.

For each $b \in M$, we define a topological metric on $P_{b}^{n}$ which depends on a given Riemannian metric $g$ on $E$. We introduce this metric to facilitate the use of the "cross ratio" discussed in the next section.

Given two nonzero vectors, $x, y \in R_{b}^{n+1}$, let $y^{\perp}$ denote the unique vector (up to sign) of the subspace determined by $x$ and $y$ such that $g\left(y, y^{\perp}\right)=0$ and $g\left(y^{\perp}, y^{\perp}\right)=g(y, y)$. Define $\rho_{b}$ on $P_{b}^{n} \times P_{b}^{n}$ by

$$
\rho_{b}([x],[y])=\frac{\left|g\left(x, y^{1}\right)\right|}{g(x, x)^{1 / 2} g(y, y)^{1 / 2}}
$$

where $|\cdot|$ denotes the absolute value of a real number. $\rho_{b}$ is a bounded metric (by 1) on $P_{b}^{n}$ giving the same topology which $P_{b}^{n}$ inherits as a subspace of $P E$. $P E$ is metrizable; let $d$ denote a metric on $P E$ giving its topology. Then $d$ and $\rho$ are related in the following way:

Proposition 2.2. For each $\epsilon>0$, the number $\delta(d, \epsilon) \equiv \inf \left\{\rho_{b}(z, w)\right\}$ $b \in M$ and $z, w \in P_{b}^{n}$ and $\left.d(z, w) \geqslant \epsilon\right\}$ is greater than zero. And, for each $\delta>0$, the number $\epsilon(\rho, \delta) \equiv \inf \left\{d(z, w) \mid \exists b \in M \ni z, w \in P_{b}^{n}\right.$ and $\rho_{b}(z, w)$ $\geqslant \delta\}$ is greater than zero.

Proof. Use the compactness of $M$ and $P E$ and the fact that $\rho$ is continuous as a function of its three arguments, $b, z$, and $w$.

COROllary 2.3. Let $b \in M$ and $z, w \in P_{b}^{n}$. Then $\lim _{t \rightarrow \infty} \rho_{b \cdot t}(z \cdot t, w \cdot t) \cdot$ $=0$ if and only if $\lim _{t \rightarrow \infty} d(z \cdot t, w \cdot t)=0$.

A statement analogous to Corollary 2.3 for $t \rightarrow-\infty$ also holds. Corollary 
2.3 asserts that if two orbits of the flow $P F$, starting in the same fiber, come together in the $\rho$ metric as $t \rightarrow \infty$ then they come together in the $d$ metric, and vice versa. Henceforth, the terminology " $z$ goes to $w$ as $t \rightarrow \infty$ " will mean the orbits of $z$ and $w$ come together as $t \rightarrow \infty$ as in Corollary 2.3.

3. Cross ratio on $P^{1}$ and $P E$. In order to exploit the linearity of $P F$, we will introduce a tool which, in local coordinates, is the cross ratio of four points in a projective line. First, consider the Euclidean plane $R^{2}$ and the associated projective line $P^{\mathbf{1}}$.

Take a basis for $R^{2}$ and let $x$ denote the column vector representation of a point in $R^{2}$ with respect to this basis. Let $\{x, y\}$ denote the $2 \times 2$ matrix with columns the vectors $x$ and $y$.

Definition. Given $[x],[y],[v],[w]$ distinct points in $P^{1}$, define the cross ratio of $[x],[y],[v],[w]$ by

$$
C([x],[y],[v],[w]) \equiv \frac{\operatorname{det}\{x, y\}}{\operatorname{det}\{x, w\}} \frac{\operatorname{det}\{v, w\}}{\operatorname{det}\{v, y\}} .
$$

REMARK. The cross ratio is independent of the basis used to define it, because if two vector representations by different bases differ by a nonsingular matrix $A$, then

$$
\frac{\operatorname{det}\{x, y\}}{\operatorname{det}\{x, w\}}=\frac{\operatorname{det} A}{\operatorname{det} A} \cdot \frac{\operatorname{det}\{x, y\}}{\operatorname{det}\{x, w\}}=\frac{\operatorname{det}\{A \cdot\{x, y\}\}}{\operatorname{det}\{A \cdot\{x, w\}\}}=\frac{\operatorname{det}\{A x, A y\}}{\operatorname{det}\{A x, A w\}} .
$$

Proposition 3.1. Let $T$ be a linear transformation on $R^{2}$ and $P T$ be the induced map on $P^{1}$. Then the cross ratio is invariant under $P T$, i.e., for distinct points $[x],[y],[v],[w] \in P^{1}$

$$
C([x],[y],[v],[w])=C([T x],[T y],[T v],[T w]) .
$$

Proof. Use the preceding computation where $A$ is the matrix of $T$.

Given an inner product $\langle\cdot, \cdot\rangle$ on $R^{2}$ and representing vectors in terms of an orthonormal basis, we see that the cross ratio can be written as (suppressing $[\cdot])$ :

$$
C(x, y, v, w)=\frac{\langle x, J y\rangle}{\langle x, J w\rangle} \frac{\langle v, J w\rangle}{\langle v, J y\rangle},
$$

where $J$ is the $2 \times 2$ matrix $\left(\begin{array}{rr}0 & 1 \\ -1 & 0\end{array}\right)$. For each positive definite, symmetric, bilinear map $g$ on $R^{2}$, there is a matrix $G$ so that $g(x, y)=\langle x, G y\rangle$ for all $x, y \in R^{2}$. The following two results say that we can express the cross ratio in terms of $g$.

LEMMA 3.2. Given a positive definite, symmetric, bilinear map $g$ on $R^{2}$ and an orthonormal basis with respect to $\langle\cdot, \cdot\rangle$, let $G$ be the matrix for $g$ in this basis. Then there are only two $2 \times 2$ matrices $J_{G}= \pm \sqrt{\operatorname{det} G} G^{-1} J$ so that 
(1) $\left\langle J_{G} x, G J_{G} y\right\rangle=\langle x, G y\rangle$ for all $x, y \in R^{2}$, and

(2) $\left\langle x, G J_{G} x\right\rangle=0$ for all $x \in R^{2}$.

Proof. (1) implies $J_{G}^{T} G J_{G}=G$ and, after taking det of both sides, we see that $\operatorname{det} J_{G}= \pm 1$. (2) implies that $G J_{G}$ is antisymmetric. Since the dimension of $2 \times 2$ antisymmetric matrices is $1, G J_{G}=\lambda J$ for some $\lambda \in R$. Since $\operatorname{det} G>0$, it follows that $\operatorname{det} J_{G}>0$. Thus $\lambda^{2}=\operatorname{det} G$.

Henceforth, $J_{G}$ will denote the matrix $\sqrt{\operatorname{det} G} G^{-1} J$. Let $x^{\prime} \equiv J_{G} x$ for all $x \in R^{2}$. Lemma 3.2 implies that for all $x, y, w \in R^{2}$ :

$$
\frac{\langle x, J y\rangle}{\langle x, J w\rangle}=\frac{\left\langle x, G J_{G} y\right\rangle}{\left\langle x, G J_{G} w\right\rangle}=\frac{g\left(x, y^{\prime}\right)}{g\left(x, w^{\prime}\right)} \text {. }
$$

Proposition 3.3. Let $g$ be any positive definite, symmetric, bilinear map on $R^{2}$. Then for distinct points $[x],[y],[v],[w] \in P^{1}$,

$$
C(x, y, v, w)=\frac{g\left(x, y^{\prime}\right)}{g\left(x, w^{\prime}\right)} \frac{g\left(v, w^{\prime}\right)}{g\left(v, y^{\prime}\right)} \text {. }
$$

REMARK. Since $x^{\prime}= \pm x^{\perp}$ for all $x \in R$

$$
|C(x, y, v, w)|=\frac{\left|g\left(x, y^{\perp}\right)\right|}{\left|g\left(x, w^{\perp}\right)\right|} \frac{\left|g\left(v, w^{\perp}\right)\right|}{\left|g\left(v, y^{\perp}\right)\right|} .
$$

Define the subset $\triangle P E$ of $P E \times P E \times P E \times P E$ by $\triangle P E \equiv\left\{(x, y, v, w) \in P E^{4} \mid \exists b \in M \ni x, y, v, w\right.$ are distinct points in the same 1-dimensional subspace of $P_{b}^{n}$.

Definition. Let $(x, y, v, w) \in \triangle P E$ with $P \pi(x)=b \in M$ and let $(\phi, U)$ be a linear bundle chart for $P E$ with $b \in U$. Then the cross ratio of $(x, y, v, w)$ is defined by

$$
C(x, y, v, w) \equiv C(\phi x, \phi y, \phi v, \phi w) .
$$

REMARK. $C$ is independent of the chart because if $(\psi, V)$ is another linear bundle chart with $b \in V$ then $C$ defined in terms of $\psi$ differs from $C$ defined in terms of $\phi$ by a linear map on $P^{1}$ (Proposition 3.1).

PROPOSITION 3.4. Let $T$ be a bundle morphism from $E$ to $E$ ( $T$ maps fibers to fibers, linearly) and $P T$ be the induced map on $P E$. Then $C$ is invariant under $P T$.

The following relates $C$ to the $\rho$ metric discussed in $\S 2$ :

Proposition 3.5. Let $g$ be a Riemannian metric on $E$ and let $\rho_{b}$ be the induced metric on $P_{b}^{n}$ for $b \in M$. Then for $(x, y, v, w) \in \triangle P E$ where $P \pi(x)=b$, 


$$
|C(x, y, v, w)|=\frac{\rho_{b}(x, y)}{\rho_{b}(x, w)} \frac{\rho_{b}(v, w)}{\rho_{b}(v, y)} .
$$

Proof. Use the remark after Proposition 3.3.

4. Notation. Unless stated otherwise, we will deal with one linear flow $(F, f)$ and one vector bundle $E$ over $M$ and its projective bundle $P E$.

The symbol $A_{1} \amalg A_{2}$ denotes the disjoint union of the disjoint sets $A_{1}$ and $A_{2}$. For a set $A$, let Int $A, \operatorname{Ext} A, \partial A, \mathrm{Cl} A$, and Comp $A$ denote the topological interior, exterior, boundary, closure, and the complement of $A$, respectively. Let $g$ be a fixed Riemannian metric on $E$ with fiber distance $\rho$, and let $d$ be the distance in the total space $P E$. If $x, y \in P^{n}$, then $\operatorname{seg}[x, y]$ will denote a closed arc from $x$ to $y$ contained in the unique $P^{\mathbf{l}}$ determined by $x$ and $y$; there are two such arcs. Open arcs will be denoted by $\operatorname{seg}(x, y)$; and half-open arcs will be denoted as usual. For compact subsets $A$ and $C$ of $E$, let $d(A, C) \equiv \inf \{d(a, c) \mid a \in A$ and $c \in C\}$; for compact $A$ contained in one fiber, let $\operatorname{diam} A \equiv \sup \{\rho(a, c) \mid a, c \in A\}$.

An isolated invariant set $S$ is a compact invariant set $(S \cdot R=S)$ which is the maximal invariant set within a closed neighborhood of itself. Maximality implies that isolated invariant sets are closed. An isolating block $B$ for $S$ is a closed set containing $S$ in its interior such that for each point $p \in \partial B$ there is an $\epsilon>0$ so that either $p \cdot(0, \epsilon) \subset \operatorname{Ext} B$ or $p \cdot(-\epsilon, 0) \subset$ Ext $B$. This definition appears different from Churchill's [2] but it is essentially the same. Every neighborhood of $S$ contains some isolating block for $S$ [2], [7], [26]. The intersection of isolated invariant sets is said to be quasi-isolated.

Except in $\S \S 10,11$, and 12, all isolated invariant sets are those of the flow $P F$.

5. How $S$ meets a 1-dimensional subspace of the fiber. Fix $b \in M$ and a 1-dimensional subspace $P_{b}^{1}$ contained in $P_{b}^{n}$. $\partial\left(S \cap P_{b}^{1}\right)$ denotes the boundary of $S \cap P_{b}^{1}$ relative to $P_{b}^{1}$. For Lemma 5.1 through Corollary 5.5, $S$ is a fixed isolated invariant set and $B$ a block for $S$.

LEMma 5.1. Suppose $Y$ is a connected subset of Int $B$ and $Y \cdot T \subset$ Int $B$ for some $T>0$. If $Y \cap S \neq \varnothing$, then $Y \cdot[0, T] \subset$ Int $B$.

Proof. Let $A \equiv\{z \in Y \mid z \cdot[0, T] \subset$ Int $B\} . A \neq \varnothing$ since $Y \cap S \neq \varnothing$; and $A$ is open by the continuity of the flow. $A$ is closed: Suppose $z_{i} \rightarrow z$ as $i \rightarrow \infty$ where $z_{i} \in A$ but $z \notin A$. There is a $t, 0<t<T$, so that $z \cdot t$ $\notin$ Int $B$. If $z \cdot t \in$ Ext $B$ then continuity implies $z_{i} \cdot t \in$ Ext $B$ for large $i$. This is a contradiction. If $z \cdot t \in \partial B$ then for some $s, 0<t+s<T, z \cdot(t+s)$ $\in$ Ext $B$. Thus $z_{i} \cdot(t+s) \in$ Ext $B$ for large $i$. 
Thus $A=Y$ since $Y$ is connected.

Definition. Let $\gamma$ be an arc in $P_{b}^{1}$ and $z \in S \cap \gamma$. Then $\gamma$ is expanding positively (negatively) at $z$ relative to $B$ if given any connected neighborhood $\gamma^{\prime}$ of $z$ relative to $\gamma$, there is a $T$ so that for all $t \geqslant T(t \leqslant T), \gamma^{\prime} \cdot t$ $\not \subset$ Int $B$.

Lemma 5.2. Let $z \in \partial\left(S \cap P_{b}^{1}\right)$. Then there is an arc $\gamma$ in $P_{b}^{1}$ so that $z$ is a boundary point of $S \cap \gamma$ relative to $\gamma$. Furthermore, any such $\gamma$ is either expanding positively or negatively at $z$ relative to $B$.

Proof. For the first assertion any open arc in $P_{b}^{1}$ containing $z$ will do. If the second assertion is false, there are two connected neighborhoods, $\gamma_{1}$ and $\gamma_{2}$, of $z$ and sequences $t_{i} \rightarrow \infty$ and $s_{i} \rightarrow-\infty$ so that $\gamma_{1} \cdot t_{i} \subset \operatorname{Int} B$ and $\gamma_{2} \cdot s_{i} \subset$ Int $B$ for all $i$. Applying Lemma 5.1, we see that $\left(\gamma_{1} \cap \gamma_{2}\right) \cdot R$ $C$ Int $B$. So $\gamma_{1} \cap \gamma_{2}$ is contained in $S$, which contradicts $z$ being a boundary point of $S \cap \gamma$ relative to $\gamma$.

DEFINITION. $z \in P_{b}^{1}$ is a positive (negative) expansion point if for every compact subset $K$ of $P_{b}^{1}-z$, diam $(K \cdot t) \rightarrow 0$ as $t \rightarrow \infty(-\infty)$.

REMARK. Consequently, if $z \in P_{b}^{1}$ is a positive expansion point then $x$ goes to $y$ as $t \rightarrow \infty$ for all $x, y \in P_{b}^{1}$ and $x, y \neq z$.

LEMMA 5.3. $P_{b}^{1}$ contains at most one positive (negative) expansion point.

PROof. Suppose $P_{b}^{1}$ contains two positive expansion points; then there is a cover of $P_{b}^{1}$ by two compact sets $K_{1}$ and $K_{2}$ so that diam $\left(K_{i} \cdot t\right) \rightarrow 0$ as $t \rightarrow \infty, i=1,2$. Thus $\operatorname{diam}\left(P_{b}^{1} \cdot t\right) \rightarrow 0$ as $t \rightarrow \infty$, which is a contradiction.

COROLlaRY 5.4. In $P_{b}^{1}$, there are five possible arrangements for expansion points. Either there is:

(1) two distinct expansion points, one positive and the other negative, or

(2) a point which is both a positive and a negative expansion point, or

(3) a positive expansion point, or

(4) a negative expansion point, or

(5) no expansion points.

Lemma 5.5. Let $\gamma$ be an arc in $P_{b}^{1}$ and $z \in S \cap \gamma$. If $\gamma$ is expanding positively (negatively) at $z$ relative to $B$, then $z$ is a positive (negative) expansion point.

Proof. Take $K$ to be a compact subset of $P_{b}^{1}-z$. Define $2 a \equiv \rho(K, z)$ and $c \equiv d(\partial B, S)$. Let $\delta \equiv \delta(d, c)$ as in Proposition 2.2; so, if $y \notin \operatorname{Int} B$, then $\rho(x, y) \geqslant \delta$ for any $x$ in $S$ and in the fiber of $y$. 
Given $\epsilon>0$ we will show there is a $T>0$ so that for all $t \geqslant T$, $\rho\left(z_{1} \cdot t, z_{2} \cdot t\right)<\epsilon$ for all $z_{1}, z_{2} \in K$. Consider a connected neighborhood $\gamma^{\prime}$ of $z$ relative to $\gamma$ so that:

(1) $\rho(z, y)<a^{2} \delta \epsilon$ for all $y \in \gamma^{\prime}$, and

(2) $\rho\left(\mathrm{Cl} \gamma^{\prime}, K\right) \geqslant a$.

Since $\gamma$ is expanding at $z$, there is a $T>0$ so that $\gamma^{\prime} \cdot t \not \subset$ Int $B$ for all $t \geqslant T$. Thus, for each $t \geqslant T$ there is some $x \in \gamma^{\prime}$ so that $x \cdot t \notin \operatorname{Int} B$, i.e., $\rho(z \cdot t, x \cdot t) \geqslant \delta$. For $z_{1}, z_{2} \in K$, the invariance of the cross ratio gives $C\left(z_{1} \cdot t, z_{2} \cdot t, z \cdot t, x \cdot t\right)=C\left(z_{1}, z_{2}, z, x\right)$. So

$$
\rho\left(z_{1} \cdot t, z_{2} \cdot t\right)=\frac{\rho\left(z_{1}, z_{2}\right)}{\rho\left(z_{1}, x\right)} \frac{\rho(z, x)}{\rho\left(z, z_{2}\right)} \frac{\rho\left(z \cdot t, z_{2} \cdot t\right)}{\rho(z \cdot t, x \cdot t)} \rho\left(z_{1} \cdot t, x \cdot t\right) .
$$

This gives $\rho\left(z_{1} \cdot t, z_{2} \cdot t\right) \leqslant \rho(z, x) / a^{2} \delta<a^{2} \delta \epsilon / a^{2} \delta=\epsilon$. Thus $\operatorname{diam}(K \cdot t)<\epsilon$ for all $t \geqslant T$.

Corollary 5.6. There are two points $x, y \in P_{b}^{1}$ such that if $S$ is an isolated invariant set then $\partial\left(S \cap P_{b}^{1}\right) \subset\{x, y\}$.

Proof. A point in $\partial\left(S \cap P_{b}^{1}\right)$ for some $S$ is a positive or negative expansion point by Lemma 5.2 and Lemma 5.5. Lemma 5.3 implies that there are at most two such points.

DEFinition. Let $P_{b}^{k}$ be a $k$-dimensional subspace of $P_{b}^{n} . G\left(P_{b}^{k}\right)$ is the set of subsets of $P_{b}^{k}$ defined as follows: $G \in G\left(P_{b}^{k}\right)$ if and only if $G$ is a connected component of $S \cap P_{b}^{k}$ for some isolated invariant set $S$.

Corollary 5.7. $G\left(P_{b}^{1}\right)$ contains at most six sets: two points, two closed arcs, $P_{b}^{1}$, and $\varnothing$.

Proof. The sets in $G\left(P_{b}^{1}\right)$ depend on the possible arrangements of positive and negative expansion points of $P_{b}^{1}$. If Corollary 5.4(1) applies, where $x$ and $y$ denote the distinct expansion points, then $G\left(P_{b}^{1}\right)$ is at most comprised of the sets: $\{x\},\{y\}$, both seg $[x, y]$ 's, $P_{b}^{1}$, and $\varnothing$. Corollary 5.4(2), (3) and (4) imply that $G\left(P_{b}^{1}\right)$ contains at most a point, $P_{b}^{1}$, and $\varnothing$. Corollary 5.4(5) implies that $G\left(P_{b}^{1}\right)$ contains at most $P_{b}^{1}$ and $\varnothing$.

The remaining results in this section are used in $\S 6$ but they pertain to a 1-dimensional subspace of the fiber.

CoRollary 5.8. Suppose a point $x$ is a component of $S \cap P_{b}^{1}$ and, for some $y \neq x, y$ goes to $x$ for a sequence $t_{m} \rightarrow \infty$. Then $x$ is a negative expansion point.

Proof. Since $y$ goes to $x$ as $t_{m} \rightarrow \infty$, Lemma 5.1 implies that there is a $T>0$ so that for one arc from $x \cdot T$ to $y \cdot T$, call it $\operatorname{seg}[x \cdot T, y \cdot T]$, 
$\operatorname{seg}[x \cdot T, y \cdot T] \cdot[T, \infty) \subset \operatorname{Int} B$. So $\operatorname{seg}[x \cdot T, y \cdot T]$ satisfies the hypothesis of Lemma 5.2 but is not expanding positively at $x \cdot T$. Thus $\operatorname{seg}[x \cdot T, y \cdot T]$ must be expanding negatively at $x \cdot T$ relative to $B$ and so $x \cdot T$ is a negative expansion point by Lemma 5.5 .

Corollary 5.9. Given $P_{b}^{k} \subset P_{b}^{n}$, suppose $G_{1}$ and $G_{2}$ are disjoint sets in $G\left(P_{b}^{k}\right)$. If $x \in G_{1}$ and $y \in G_{2}$, then the orbits of $x$ and $y$ are bounded apart for all $t \in R$.

Proof. Suppose $x$ goes to $y$ for a sequence $t_{m} \rightarrow \infty$. Let $P_{b}^{1}$ be the 1-dimensional subspace determined by $x$ and $y$. Since $G_{1}$ and $G_{2}$ are disjoint, $x=S_{1} \cap P_{b}^{1}$ and $y=S_{2} \cap P_{b}^{1}$ for isolated invariant sets $S_{1}$ and $S_{2}$ (Corollary 5.7). Corollary 5.8 implies that both $x$ and $y$ are negative expansion points, which is a contradiction. A similar argument shows that $x$ cannot go to $y$ for a sequence $t_{m} \rightarrow-\infty$.

COROLlaRY 5.10. Given $S$ isolated, suppose there are two distinct points in $\partial\left(S \cap P_{b}^{1}\right)$. For the positive expansion point $x$, there is an open arc $\gamma$ containing $x$ so that $\gamma \cdot t \subset$ Int $B$ for all $t \leqslant 0$; and for the negative expansion point $y$, there is an open arc $\sigma$ containing $y$ so that $\sigma \cdot t \subset$ Int $B$ for all $t \geqslant 0$.

Proof. Since $y$ is a negative expansion point, there is an open arc $\gamma$ so that $x \in \gamma \subset$ Int $B$ and $\operatorname{diam}(\gamma \cdot t) \rightarrow 0$ as $t \rightarrow-\infty$. So Lemma $5.1 \mathrm{im}$ plies $\gamma \cdot(-\infty, 0] \subset$ Int $B$. Do likewise for some $\sigma$ containing $y$.

LEMMA 5.11. Suppose $S \cap P_{b}^{1}$ contains more than one point. Let $x \in$ $S \cap P_{b}^{1}$ and $y \in P_{b}^{1}$ with $y \cdot[0, \infty) \subset$ Int $B$. If one of the arcs from $x$ to $y$, call it $\operatorname{seg}[x, y]$, is contained in Int $B$, then $\operatorname{seg}[x, y] \cdot[0, \infty) \subset$ Int $B$.

Proof. Assume $\operatorname{seg}[x, y]$ contains a point in $\partial\left(S \cap P_{b}^{1}\right)$; otherwise $\operatorname{seg}[x, y] \subset S$ and we are done. So, without loss of generality, assume $x$ is the closest point in $\partial\left(S \cap P_{b}^{1}\right) \cap \operatorname{seg}[x, y)$ to $y$ and assume $\operatorname{seg}(x, y) \cap S=\varnothing$.

Case 1. $y \notin \partial\left(S \cap P_{b}^{1}\right)$. So there is a $z \notin \operatorname{seg}[x, y]$ so that $z \in \partial\left(S \cap P_{b}^{1}\right)$. If $z$ is a positive expansion point then $\operatorname{diam}(\operatorname{seg}[x, y] \cdot t) \rightarrow 0$ as $t \rightarrow \infty$, and Lemma 5.1 implies our conclusion. But $z$ cannot be a negative expansion point because then $y \cdot R \subset \operatorname{Int} B$ which contradicts $y \notin \partial\left(S \cap P_{b}^{1}\right)$.

Case 2. $y \in \partial\left(S \cap P_{b}^{1}\right)$. If $y$ is a positive expansion point then, for each $w \in \operatorname{seg}(x, y), \operatorname{seg}[x, w] \cdot[0, \infty) \subset \operatorname{Int} B$ so we are done. If $x$ is a positive expansion point then, for each $w \in \operatorname{seg}(x, y), \operatorname{seg}[y, w] \cdot[0, \infty) \subset$ Int $B$.

6. Preliminary lemmas for the fiber. Again fix $b \in M$ and $P_{b}^{k}$, a $k$-dimensional subspace of $P_{b}^{n}$ where $k \leqslant n$. For all results in this section, $S$ is a 
fixed isolated invariant set. We begin with a lemma which depends only on the geometry of $n$-dimensional projective space.

Lemma 6.1. Let $b \in M$ and $x \neq y \in P_{b}^{n}$. Given $\operatorname{seg}[x, y]$ and $a \delta$, $0<\delta<1 / 2$, then there exists a neighborhood $U$ of $y$ in $P_{b}^{n}$ so that for each $z \in U$ there is a unique $\operatorname{seg}[x, z]$ within $\delta$ of $\operatorname{seg}[x, y]$, i.e., $\rho(w, \operatorname{seg}[x, y])$ $<\delta$ for all $w \in \operatorname{seg}[x, z]$ and $\rho(w, \operatorname{seg}[x, z])<\delta$ for all $w \in \operatorname{seg}[x, y]$. Furthermore, the union over $z \in U$ of $\operatorname{seg}(x, z]$ is a neighborhood of $\operatorname{seg}(x, y]$ in $P_{b}^{n}$.

REMARK. Such a neighborhood of $\operatorname{seg}(x, y]$ is called a cone-like neighborhood of $\operatorname{seg}(x, y]$ over $U$.

LEMma 6.2. Given $S$, there exists some $\delta>0$ so that if $x, y \in S \cap P_{b}^{n}$ and $\rho(x, y)<\delta$ then the $\operatorname{seg}[x, y]$ of $\rho$-length $<\delta$ is contained in $S$. (The same $\delta$ works for all $b \in M$.)

Proof. Take a block $B$ for $S$. Let $c \equiv d(\partial B, S)$. Define $\delta \equiv \delta(d, c)$. If $\rho(x, y)<\delta$ for $x, y \in S \cap P_{b}^{n}$, then $\rho(z, x)<\delta$ for all $z \in \operatorname{seg}[x, y]$. Thus $\operatorname{seg}[x, y] \subset$ Int $B$. Lemma 5.11 implies that $\operatorname{seg}[x, y] \cdot R \subset$ Int $B$ and so $\operatorname{seg}[x, y] \subset S$.

COROLlaRY 6.3. $S \cap P_{b}^{n}$ has at most a finite number of connected components.

Proof. Each component must be separated by the $\delta$ in Lemma 6.2.

Definition. A subset $A$ of $P_{b}^{k}$ is arc connected if for each pair $x \neq y$ $\in A$ at least one $\operatorname{seg}[x, y]$ is contained in $A$.

LEMmA 6.4. If $S \cap P_{b}^{k}$ is connected then $S \cap P_{b}^{k}$ is arc connected.

Proof. Take a block $B$ for $S$. Let $c \equiv d(\partial B, S)$ and $\delta \equiv \delta(d, c)$. Fix $x \in S \cap P_{b}^{k}$ and define $A \equiv\left\{y \in S \cap P_{b}^{k} \mid\right.$ at least one $\left.\operatorname{seg}[x, y] \subset S \cap P_{b}^{k}\right\}$. $A \neq \varnothing$ since $S \cap P_{b}^{k}$ is connected and Lemma 6.2 implies that points of $S \cap P_{b}^{k}$ close to $x$ are connected to $x$ by arcs in $S \cap P_{b}^{k}$.

$A$ is open: Lemma 6.2 implies that $x \in \operatorname{Int} A$. Take $y \neq x \in A$ and $\operatorname{seg}[x, y] \subset S \cap P_{b}^{k}$. Use Lemma 6.1 to get a neighborhood $U$ of $y$ so that $\operatorname{seg}[x, z]$ is within $\delta$ of $\operatorname{seg}[x, y]$ for all $z \in U$. Let $W \equiv U \cap\left(S \cap P_{b}^{k}\right)$. For each $w \in W, \operatorname{seg}[x, w] \subset$ Int $B$; and this gives $\operatorname{seg}[x, w] \cdot R \subset$ Int $B$ by Lemma 5.11. Thus $w \in A$ and so $W \subset A$.

$A$ is closed: Take $y_{i} \in A$ such that $y_{i} \rightarrow y$ as $i \rightarrow \infty$ where $y \in S$ $\cap P_{b}^{k}$. Without loss of generality, assume $\operatorname{seg}\left[x, y_{i}\right]$ converges to one $\operatorname{seg}[x, y]$ as $i \rightarrow \infty$. Thus, for large $i, \operatorname{seg}[x, y]$ is within $\delta$ of $\operatorname{seg}\left[x, y_{i}\right] \subset S \cap P_{b}^{k}$. So $\operatorname{seg}[x, y] \subset$ Int $B$ and, as above, $y \in A$. 
Therefore, $A=S \cap P_{b}^{k}$ since $S \cap P_{b}^{k}$ is connected.

Definition. $\left[x_{1}\right],\left[x_{2}\right], \cdots,\left[x_{k}\right] \in P_{b}^{n}$ are linearly independent if $x_{1}$, $x_{2}, \cdots, x_{k} \in R_{b}^{n+1}$ are linearly independent.

LEMma 6.5. If $S \cap P_{b}^{k}$ is connected and contains $k+1$ linearly independent points, then $S \cap P_{b}^{k}$ has interior relative to $P_{b}^{k}$.

Proof. Let $x_{1}, x_{2}, \cdots, x_{k+1}$ be $k+1$ linearly independent points in $S \cap P_{b}^{k}$.

Claim. For $r \leqslant k$, Int $\left(S \cap P_{b}^{r}\right)$ relative to $P_{b}^{r}$ is nonempty, where $P_{b}^{r}$ is the $r$-dimensional subspace of $P_{b}^{k}$ spanned by $\left\{x_{1}, \cdots, x_{r+1}\right\}$. We argue by induction on $r$. For $r=0$, the claim is obvious. Assume it is true for the $P_{b}^{r-1}$ spanned by $\left\{x_{1}, \cdots, x_{r}\right\}$ and consider the $P_{b}^{r}$ spanned by $\left\{x_{1}, \cdots, x_{r+1}\right\}$. Let $y \in \operatorname{Int}\left(S \cap P_{b}^{r-1}\right)$ relative to $P_{b}^{r-1}$. By Lemma 6.4 one $\operatorname{seg}\left[x_{r+1}, y\right]$ is contained in $S \cap P_{b}^{r}$. Take a block $B$ for $S$ and let $\delta \equiv \delta(d, c)$ where $c=$ $d(\partial B, S)$. Construct a cone-like neighborhood within $\delta$ of $\operatorname{seg}\left[x_{r+1}, y\right]$ over a neighborhood of $y$ in $\operatorname{Int}\left(S \cap P_{b}^{r-1}\right)$ relative to $P_{b}^{r-1}$. Applying Lemma 5.11 , we see that this cone-like neighborhood of $\operatorname{seg}\left(x_{r+1}, y\right]$ is contained in Int $\left(S \cap P_{b}^{r}\right)$ relative to $P_{b}^{r}$.

LEMma 6.6. Suppose $S \cap P_{b}^{k}$ is connected and contains $k+1$ linearly independent points. If $k>1$, then $\partial\left(S \cap P_{b}^{k}\right)$ relative to $P_{b}^{k}$ is connected.

Proof. Let $A \equiv S \cap P_{b}^{k}$. $A$ being arc connected (Lemma 6.4) implies that $\operatorname{Comp}\left(S \cap P_{b}^{k}\right)$ is arc connected. Let $A^{\prime} \equiv \mathrm{Cl}\left(\operatorname{Comp}\left(S \cap P_{b}^{k}\right)\right)$ relative to $P_{b}^{k}$.

In Čech homology every compact triad is proper [12], so $\left(P_{b}^{k}, A, A^{\prime}\right)$ is proper. Appealing to the first four terms of the reduced Mayer-Vietoris sequence in Cech homology with real coefficients, we get the exact sequence:

$$
H_{1}\left(A \cup A^{\prime}\right) \rightarrow H_{0}\left(A \cap A^{\prime}\right) \rightarrow H_{0}(A) \oplus H_{0}\left(A^{\prime}\right) \rightarrow H_{0}\left(A \cup A^{\prime}\right)
$$

which is

$$
H_{1}\left(P_{b}^{k}\right) \rightarrow H_{0}\left(\partial\left(S \cap P_{b}^{k}\right)\right) \rightarrow H_{0}(A) \oplus H_{0}\left(A^{\prime}\right) \rightarrow H_{0}\left(P_{b}^{k}\right) .
$$

Since $A$ and $A^{\prime}$ are connected, $H_{0}(A)=H_{0}\left(A^{\prime}\right)=0$; and, since $k>1$, $\dot{H}_{1}\left(P_{b}^{k}\right)=0$. Thus $H_{0}\left(\partial\left(S \cap P_{b}^{k}\right)\right)=0$ so $\partial\left(S \cap P_{b}^{k}\right)$ is connected.

LEMMA 6.7. Suppose $S \cap P_{b}^{k}$ is connected and contains $k+1$ linearly independent points. If $k>1$, then $S \cap P_{b}^{k}=P_{b}^{k}$.

Proof. If not, then $\partial\left(S \cap P_{b}^{k}\right)$ relative to $P_{b}^{k}$ is not empty.

Take a block $B$ for $S$ with $\delta \equiv \delta(d, c)$ where $c=d(\partial B, S)$. Fix $x \in$ $\partial\left(S \cap P_{b}^{k}\right)$ relative to $P_{b}^{k}$. For any $y \in \operatorname{Int}\left(S \cap P_{b}^{k}\right)$ relative to $P_{b}^{k}$, one $\operatorname{seg}[x, y]$ is contained in $S \cap P_{b}^{k}$ by Lemma 6.4. For the $P_{b}^{1}$ spanned by $x$ 
and $y$, it is true that $x \in \partial\left(S \cap P_{b}^{1}\right)$ relative to this $P_{b}^{1}$. If not, we can extend $\operatorname{seg}[x, y]$ to $\operatorname{seg}[z, y] \subset S \cap P_{b}^{k}$ where $x \in \operatorname{seg}(z, y)$. Constructing a conelike neighborhood for $\operatorname{seg}[z, y]$ within $\delta$ of $\operatorname{seg}(z, y]$ forces $x \in \operatorname{Int}\left(S \cap P_{b}^{k}\right)$, which is a contradiction.

By Lemma 5.5, $x$ is either a positive expansion point or a negative expansion point with respect to $y$ but not both because $\partial\left(S \cap P_{b}^{1}\right)$ contains two points. If $x$ is a positive expansion point with respect to $y$, then $x$ is a positive expansion point with respect to any other $y^{\prime} \in \operatorname{Int}\left(S \cap P_{b}^{k}\right)$ : Lemma 5.10 implies there is an $\operatorname{arc} \gamma_{y} \subset P_{b}^{1}$ so that $\gamma_{y} \cdot t \subset \operatorname{Int} B$ for all $t \leqslant 0$. For $z \in$ $\gamma_{y} \cap$ Comp $S$ and close to $x$, take a cone-like neighborhood $V$ of $\operatorname{seg}(z, y]$ over a neighborhood $U$ of $y$ in $\operatorname{Int}\left(S \cap P_{b}^{k}\right)$. Choose these neighborhoods so that, for all $w \in U$, the $\operatorname{seg}[z, w]$ in $V$ is within $\delta$ of $S \cap P_{b}^{k}$ and so is in Int $B$. Since $z \cdot(-\infty, 0] \subset$ Int $B$, Lemma 5.11 implies $V \cdot(-\infty, 0] \subset$ Int $B$. So for some other $y^{\prime} \in \operatorname{Int}\left(S \cap P_{b}^{k}\right)$, the $P_{b}^{1}$ determined by $x$ and $y^{\prime}$ meets $V$ in an open arc $\gamma_{y^{\prime}}$ which must remain in $\operatorname{Int} B$ for all $t \leqslant 0$. Thus $\gamma_{y^{\prime}}$ is expanding positively at $x$ by Lemma 5.2 and $x$ is a positive expansion point with respect to $y^{\prime}$ by Lemma 5.5 .

Define $A \equiv\left\{x \in \partial\left(S \cap P_{b}^{k}\right) \mid x\right.$ is a positive expansion point $\}$, and $A^{\prime} \equiv$ $\left\{x \in \partial\left(S \cap P_{b}^{k}\right) \mid x\right.$ is a negative expansion point $\} . A \neq \varnothing$ implies $A^{\prime} \neq \varnothing$ since a $P_{b}^{1}$ spanned by a positive expansion point in $\partial\left(S \cap P_{b}^{k}\right)$ and a point of Int $\left(S \cap P_{b}^{k}\right)$ must contain a negative expansion point. So $A$ and $A^{\prime}$ are nonempty, disjoint sets whose union is $\partial\left(S \cap P_{b}^{k}\right) . A$ is open: The argument is the same as that showing the "positive expansion point" characterization is independent of $y \in \operatorname{Int}\left(S \cap P_{b}^{k}\right)$. Observe that the cone-like neighborhood $V$ meets $\partial\left(S \cap P_{b}^{k}\right)$ in a neighborhood of $x$, all of whose points must be positive expansion points.

Likewise $A^{\prime}$ is open. But this disconnects $\partial\left(S \cap P_{b}^{k}\right)$, and thus contradicts Lemma 6.6.

7. Main results on how $S$ meets the fiber.

THEOREM 7.1. Let $S$ be an isolated invariant set. Let $P_{b}^{k}$ denote some $k$-dimensional subspace of $P_{b}^{n}, 0 \leqslant k \leqslant n$. Then $S \cap P_{b}^{k}=\amalg_{i=1}^{s} S \cap P_{b}^{k_{i}}, 0 \leqslant$ $k_{i} \leqslant k$, where the $P_{b}^{k_{i}}$ are disjoint $k_{i}$-dimensional subspaces of $P_{b}^{k}$ and

(1) if $k_{i} \neq 1$ then $S \cap P_{b}^{k_{i}}=P_{b}^{k_{i}}$;

(2) if $k_{i}=1$ then $S \cap P_{b}^{1}=P_{b}^{1}$ or $S \cap P_{b}^{1}$ is a closed arc of $P_{b}^{1}$ (a half-P $P^{1}$ ).

Proof. If a component of $S \cap P_{b}^{k}$ has more than two linearly independent points, take a maximal set of linearly independent points in the component and consider the $P_{b}^{k_{i}}$ they span. Lemma 6.7 implies the component is exactly this $P_{b}^{k_{i}}$. If a component of $S \cap P_{b}^{k}$ has two or less independent points, the result follows from Corollary 5.7. 
The only possibility for an intersection of these $P_{b}^{k_{i}}$,s is if they are $P_{b}^{1}$ 's containing half- $P^{1}$ components. But the point of intersection is forced to go to both components simultaneously, which is a contradiction (Lemma 6.2).

LEMma 7.2. For $0 \leqslant k \leqslant n$, let $P_{b}^{k}$ be a $k$-dimensional subspace of $P_{b}^{n}$ and let $\left\{A_{1}, \cdots, A_{r}\right\}$ be a collection of pairwise disjoint sets in $G\left(P_{b}^{k}\right)$. Then $\sum_{i=1}^{r}\left(\operatorname{dim} A_{i}+1\right) \leqslant k+1$, where the dimension of a half- $P^{1}$ is 1 . Also, if $\sum_{i=1}^{r}\left(\operatorname{dim} A_{i}+1\right)=k+1$, then each $w \in P_{b}^{k}$ goes to a point of one of these $A$ 's as $t \rightarrow \infty$ and to a point of one as $t \rightarrow-\infty$.

Proof. Use induction on $k$. The case $k=1$ follows from Corollary 5.7. Assume the result for $k-1$. To prove the first assertion for $k$, assume there is a collection $\left\{A_{1}, \cdots, A_{r}\right\}$ with $\Sigma_{i=1}^{r}\left(\operatorname{dim} A_{i}+1\right)>k+1$. By Theorem 7.1, $A_{i}=P_{b}^{k_{i}}$ except if $A_{i}$ is a half- $P^{1}$ and then it is contained in a unique $P_{b}^{1}$. For each $i$, choose $k_{i}+1$ linearly independent points in $A_{i}$ $\left(\operatorname{dim} A_{i}=k_{i}\right)$. Let $L$ denote the set of all such points. By induction, any $k+1$ points of $L$ are linearly independent.

Case 1. One $A_{i}$, say $A_{r}$, is either a half- $P^{1}$ or a point. Let $\left\{x_{1}, \cdots, x_{k+2}\right\}$ be $k+2$ points of $L$ with either $x_{k+1}, x_{k+2} \in A_{r}$ or $x_{k+2}=A_{r}$. The intersection of the $P_{b}^{k-1}$ spanned by $\left\{x_{1}, \cdots, x_{k}\right\}$ and the $P_{b}^{1}$ spanned by $x_{k+1}$ and $x_{k+2}$ contains some point $y . y$ goes to $x_{k+2}$ as $t \rightarrow \infty$ or as $t \rightarrow-\infty$. Without loss of generality, assume $y$ goes to $x_{k+2}$ as $t \rightarrow \infty$. By induction, $y$ goes to one of the first $r-1 A_{i}$ 's as $t \rightarrow \infty$. This contradicts Corollary 5.9.

Case 2. No $A_{i}$ is a half $P^{1}$ or a point. Let $\left\{x_{1}, \cdots, x_{k+2}\right\}$ be $k+2$ points of $L$ where $x_{1}$ and $x_{2}$ belong to the same $A_{i}$, say $A_{1} \cdot x_{k+2}$ is a linear combination of $\left\{x_{1}, \cdots, x_{k+1}\right\}$. Since linear combinations of $x_{1}$ and $x_{2}$ belong to $A_{1}, x_{k+2}$ is a linear combination of $k$ independent points $\left\{a_{1} x_{1}+a_{2} x_{2}, x_{3}, \cdots, x_{k+1}\right\}$ where $a_{1}, a_{2} \in R$. This contradicts the induction hypothesis.

To prove the second assertion, let $\sum_{i=1}^{r}\left(\operatorname{dim} A_{i}+1\right)=k+1$ and take $L=\left\{x_{1}, \cdots, x_{k+1}\right\}$ as above. $\left\{x_{1}, \cdots, x_{k+1}\right\}$ are linearly independent. By induction, we may assume that $w$ is a linear combination of all $x_{1}, \cdots, x_{k+1}$. The $P_{b}^{1}$ spanned by $w$ and $x_{k+1}$ meets the $P_{b}^{k-1}$ spanned by $x_{1}, \cdots, x_{k}$ in a point $y_{1}$. By induction, $y_{1}$ goes to one of the first $k A$ 's as $t \rightarrow \infty$ and to one as $t \rightarrow-\infty$. Without loss of generality, assume $x_{k+1}$ is a negative expansion point (if $x_{k+1} \notin \partial\left(S \cap P_{b}^{1}\right.$ ) for some $S$ isolated then $P_{b}^{1}$ contains two points in $\partial\left(S \cap P_{b}^{1}\right)$ and we are done). Thus $w$ goes to $y_{1}$ as $t \rightarrow \infty$ and so to a point of one of the $A$ 's as $t \rightarrow \infty$, say $x_{1}$. Now take the $P_{b}^{1}$ spanned by $w$ and $x_{1}$. Again assume $x_{1}=\partial\left(S \cap P_{b}^{1}\right)$ for some $S$. 
$P_{b}^{1}$ meets the $P_{b}^{k-1}$ spanned by $x_{2}, x_{3}, \cdots, x_{k+1}$ in a point $y_{2}$. By induction, $y_{2}$ goes to the $A$ 's as $t \rightarrow \infty$ and as $t \rightarrow-\infty$. Since $w$ goes to $x_{1}$ as $t \rightarrow \infty, w$ goes to $y_{2}$ as $t \rightarrow-\infty$ (Corollary 5.8). Thus $w$ goes to a point in one of the $A$ 's as $t \rightarrow-\infty$.

THEOREM 7.3. Let $S$ be an isolated invariant set and $P_{b}^{k}$ be a k-dimensional subspace of $P_{b}^{n}, 0 \leqslant k \leqslant n$. Suppose $S \cap P_{b}^{k}=\amalg_{i=1}^{S} P_{b}^{k_{i}}, 0 \leqslant k_{i} \leqslant k$, where $P_{b}^{1}$ may denote a half- $P^{1}$ (Theorem 7.1). Then $\Sigma_{i=1}^{s}\left(k_{i}+1\right) \leqslant k+1$.

Proof. This is a consequence of Lemma 7.2.

THEOREM 7.4. For any k-dimensional subspace $P_{b}^{k}$ of $P_{b}^{n}$, let $D\left(P_{b}^{k}\right)$ denote the set of subspaces of $P_{b}^{k}$ such that $G \in D\left(P_{b}^{k}\right)$ if $G$ is a component of $S \cap P_{b}^{k}$ for some isolated invariant set $S$ ( $D$ is $G$ minus half $-P_{b}^{1}$ 's). Then $D\left(P_{b}^{k}\right)$ is finite.

Proof. $D\left(P_{b}^{k}\right)=\bigcup_{m=0}^{k} D_{m}\left(P_{b}^{k}\right)$ where $D_{m}\left(P_{b}^{k}\right)$ is the set.of $m$-dimensional subspaces in $D\left(P_{b}^{k}\right)$. We proceed by induction on $k$. For $k=1$ and $m=0,1$, Corollary 5.7 applies. So we assume the assertion for $k-1$. To prove it for $k$ we use induction on $m$.

For $m=0$, the result follows from Lemma 7.2. Assume $D_{m-1}\left(P_{b}^{k}\right)$ is finite. Consider a $(k-1)$-dimensional subspace $P_{b}^{k-1}$ of $P_{b}^{k}$. By induction, $D_{m}\left(P_{b}^{k-1}\right)$ is finite, so we will show the $m$-dimensional subspaces in $D_{m}\left(P_{b}^{k}\right)$ which are not subsets of $P_{b}^{k-1}$ are finite in number. By induction, $D_{m-1}\left(P_{b}^{k-1}\right)=$ $\left\{A_{1}, \cdots, A_{r}\right\}$. All subspaces in $D_{m}\left(P_{b}^{k}\right)-D_{m}\left(P_{b}^{k-1}\right)$ must meet $P_{b}^{k-1}$ in one of these $A$ 's. But for each $A_{i}, i=1, \cdots, r$, there are only a finite number of subspaces $G \in \mathcal{D}_{m}\left(P_{b}^{k}\right)$ so that $G=A_{i} \cap P_{b}^{k-1}$ : Take $x \in A_{i}$ and a $\widetilde{P}_{b}^{k-1}$ complementary to $x$ in $P_{b}^{k}$. Each such $G$ must meet $\widetilde{P}_{b}^{k-1}$ in a different set in $D_{m-1}\left(\widetilde{P}_{b}^{k-1}\right)$. And $D_{m-1}\left(\widetilde{P}_{b}^{k-1}\right)$ is finite by induction.

Thus $D_{m}\left(P_{b}^{k}\right)-D_{m}\left(P_{b}^{k-1}\right)$ is finite. So $D_{m}\left(P_{b}^{k}\right)$ is finite.

8. Morse sets and chain recurrence. In this section and the next, we restrict our attention to a subset of the set of isolated invariant sets. These sets are called Morse sets because the generalized Morse inequalities apply [5]. The following discussion makes use of results in an I. B. M. research paper, Gradient structure of a flow. I (RC 3932, July 12, 1972), by C. Conley.

For a flow $f$ on a compact metric space $(M, d)$, let $f^{*}$ denote the backwards flow defined by $f_{t}^{*}(x)=f_{-t}(x)$. For $Y \subset M$, let $\omega(Y, f)$ denote $\bigcap_{t>0} \mathrm{Cl}\{Y \cdot(t, \infty)\}$ and be called the $\omega$-limit set of $Y$. The $\alpha$-limit set of $Y$ is $\omega\left(Y, f^{*}\right)$. A subset $A$ is called an attractor for $f$ if $A$ has a closed neighborhood $N$ so that $A=\omega(N, f)$. A quasi-attractor is the intersection of attractors. An attractor for $f^{*}$ is called a repeller for $f$. Let $A^{*} \equiv \operatorname{Comp}\{x \mid \omega(x, f) \subset A\}$. 
LEMMA 8.1. If $A \neq M$ and is an attractor for $f$, then $A^{*}$ is nonempty and is a repeller for $f$.

Remark. $A^{*}$ is called the repeller for $f$ dual to $A$. The repeller for $f^{*}$ dual to $A^{*}$ is exactly $A$.

We may define a generalized limit set and recurrent set in terms of the attractors of $f$. For any subset $Y$, let $\Omega(Y, f)$ be the intersection of all attractors of $f$ containing $\omega(Y, f)$, and define $R(f)$ to be the intersection, over all attractors $A$, of $A \cup A^{*} . R(f)$ is called the chain recurrent set of $f$. The rationale behind the terminology is that $R(f)$ can be characterized in terms of a recurrence relation.

Given $(x, y) \in M \times M$ and $\epsilon, t>0$, an $(\epsilon, t, f)$-chain from $x$ to $y$ is a collection $\left\{x_{1}=x, x_{2}, \cdots, x_{k+1}=y ; t_{1}, t_{2}, \cdots, t_{k}\right\}$ so that for $1 \leqslant i \leqslant n$ we have $t_{i} \geqslant t$ and $d\left(x_{i} \cdot t_{i}, x_{i+1}\right)<\epsilon$. Let $P(f) \equiv\{(x, y) \mid$ for any $\epsilon, t>0$ there exists an $(\epsilon, t, f)$-chain from $x$ to $y\} ; P(f)$ is closed in $M \times M$ and is a transitive relation.

Proposition 8.2. For $Y \subset M, \Omega(Y, f)=\{x \mid$ there exists some $y \in Y$ so that $(y, x) \in P(f)\}$. (In particular, for $x \in M, \omega(x, f) \subset \Omega(x, f)$.)

LEMMA 8.3. For $x, y \in M$, if $y \in \Omega(x, f)$ then $x \in \Omega\left(y, f^{*}\right)$.

Proposition 8.4. $R(f)=\{x \mid(x, x) \in P(f)\}$. Equivalently, $R(f)=$ $\{x \mid x \in \Omega(x, f)\}$.

The set $R(f)$ is a closed invariant set containing the nonwandering set and $R(f)=R\left(f^{*}\right)$. Roughly speaking, the connected components of $R(f)$ contain the nongradient-like behavior of $f$. In fact, if each component of $R(f)$ is identified with a point then the induced flow on the identification space is gradientlike, i.e., there are continuous functions which decrease on all nonconstant orbits of the induced flow and the set of rest points is totally disconnected. The constant orbits of the induced flow are precisely the images in the identification space of the components of $R(f)$.

Given $Y \subset M, f$ is chain recurrent on $Y$ if and only if $R\left(\left.f\right|_{Y}\right)=Y$.

Proposition 8.5. If $f$ is chain recurrent on a connected set $Y$ and if $x, y, \in Y$, then $(x, y) \in P(f)$ and $(y, x) \in P(f)$.

Proposition 8.6. If $\left.\bar{f} \equiv f\right|_{R(f)}$ then $R(\bar{f})=R(f)$.

From Proposition 8.6, it follows that $f$ is chain recurrent on $R(f)$. Proposition 8.5 implies that the components of $R(f)$ are the maximal connected subsets of $M$ on which $f$ is chain recurrent. Also, $f$ is chain recurrent on the $\alpha$ - and $\omega$-limit sets of a point and on a minimal set (a closed, invariant set which is minimal with respect to these properties). 
Definition. A Morse set is the intersection of an attractor and a repeller. A quasi-Morse set is the intersection of Morse sets. (Morse sets are isolated invariant sets since attractors and repellers are isolated and finite intersections of isolated invariant sets are isolated.)

Proposition 8.7. $S$ is a Morse set if and only if $S=\Omega(S, f) \cap \Omega\left(S, f^{*}\right)$ and $S \cap R(f)$ is open and closed in $R(f)$.

A Morse decomposition of $f$ is a finite ordered collection of Morse sets $\left\{S_{1}, S_{2}, \cdots, S_{k}\right\}$ such that

(a) $R(f)=\bigcup_{i=1}^{k} R(f) \cap S_{i}$;

(b) for $i<j, \Omega\left(S_{i}, f\right) \cap S_{j}=\varnothing$ and $\Omega\left(S_{j}, f^{*}\right) \cap S_{i}=\varnothing$.

REMARK. If $\{S\}$ is a Morse decomposition of $f$, then $S=M$ because, if not, $S=A_{1} \cap A_{2}^{*} \neq M$ where $A_{1}$ and $A_{2}$ are attractors. But then either $A_{1}^{*}$ or $A_{2}$ contains points of $R(f)$ not in $S$.

A filtration of $f$ is a finite increasing sequence $\left\{\varnothing=A_{0}, A_{1}, \cdots, A_{k}=M\right\}$ of attractors for $f$.

Proposition 8.8. There is a one-one correspondence between filtrations and Morse decompositions such that to the filtration $\left\{\varnothing=A_{0}, \cdots, A_{k}\right\}$ there corresponds the Morse decomposition $\left\{S_{1}, \cdots, S_{k}\right\}$ where $S_{i}=A_{i} \cap A_{i-1}^{*}$, $i=1, \cdots, k$. To the Morse decomposition $\left\{S_{1}, \cdots, S_{k}\right\}$ there corresponds the filtration $A_{i}=\Omega\left(S_{1} \cup \cdots \cup S_{i}, f\right), i=1, \cdots, k$; and $A_{0}=\varnothing$.

LEmma 8.9. If $S \neq M$ and is a Morse set, then there is a Morse decomposition of $f$ having at least two Morse sets with $S$ heing one of these.

Proof. By Lemma 8.1, $S=A_{1} \cap A_{2}^{*}$ where $A_{1}$ and $A_{2}$ are attractors for $f$. Since $S \neq M$, either $A_{1} \neq M$ or $A_{2} \neq \varnothing$. If $A_{1}=M$, then $S=$ $A_{2}^{*}$ and $\left\{A_{2}, A_{2}^{*}\right\}$ is a Morse decomposition. If $A_{2}=\varnothing$, then $S=A_{1}$ and $\left\{A_{1}, A_{1}^{*}\right\}$ works. And if $A_{1} \neq M$ and $A_{2} \neq \varnothing$, then $\left\{A_{2}, S, A_{1}^{*} \cap A_{2}^{*}\right\}$ works.

LEMMA 8.10. Let $\left\{S_{1}, \cdots, S_{k}\right\}$ be a Morse decomposition of $f$. Suppose $S$ is a closed invariant subset of $M$. Then $\left\{S_{1} \cap S, \cdots, S_{k} \cap S\right\}$ is a Morse decomposition of $\left.f\right|_{s}$.

9. Morse sets in projective bundles. Let us return to the situation where $E$ is an $n+1$-dimensional vector bundle over $M$ and $P E$ is its associated projective bundle. $(F, f)$ and $(P F, f)$ denote a linear flow on these bundles. The following lemma shows that a Morse set of $P F$ meets the fiber in a simpler way than does an arbitrary isolated invariant set.

Lemma 9.1. Let $S$ be a Morse set in $P E$ and $b \in M$. There is some 
$k$-dimensional subspace, where $k$ depends on $b$, so that $S \cap P_{b}^{n}=P_{b}^{k}$. (Thus $S \cap P_{b}^{n}$ has at most one component and the exceptional half-P1 cannot occur.)

Proof. Suppose $S \cap P_{b}^{n}$ has at least two components. Take a point $x$ in one component and $y$ in another component. Fix $z$ in the $P_{b}^{1}$ spanned by $x$ and $y$ and assume $z \notin S$. Corollary 5.7 implies that $S \cap P_{b}^{1}=\{x, y\}$. By Lemmas 5.2 and 5.5, either $x$ or $y$ is a positive expansion point and the other is a negative expansion point. Without loss of generality, assume $y$ is the positive expansion point. Then $z \cdot t \rightarrow x \cdot t$ as $t \rightarrow \infty$. So, for some $w \in$ $\omega(x, P F) \subset S$, there is a sequence $t_{m} \rightarrow \infty$ such that $z \cdot t_{m} \rightarrow w$. Thus $w \in \omega(z, P F)$ and so Lemma 8.3 implies that $z \in \Omega\left(w, P F^{*}\right)$. Thus $z \in$ $\Omega\left(S, P F^{*}\right)$. Likewise, for some $u \in \omega\left(y, P F^{*}\right) \subset S$, there is a sequence $s_{m} \rightarrow$ $-\infty$ so that $z \cdot s_{m} \rightarrow u$. So $z \in \Omega(u, P F) \subset \Omega(S, P F)$. But then $z \in$ $\Omega(S, P F) \cap \Omega\left(S, P F^{*}\right)=S$, which is a contradiction. $\left(S=\Omega(S, P F) \cap \Omega\left(S, P F^{*}\right)\right.$ because $S$ is a Morse set.)

The same argument shows that $S \cap P_{b}^{n}$ cannot contain a half- $P^{1}$ since, if it does, a point in the other half is forced to be in $\Omega(S, P F) \cap \Omega\left(S, P F^{*}\right)=S$.

Now we shall state and prove the splitting theorem for a Morse decomposition of $P F$ over a connected space $Q$ on which $f$ is chain recurrent. One might suspect this result by analyzing the case where $Q$ is a rest point of a $C^{\mathbf{1}}$. flow $f$ and $F_{t}=T f_{t}$, i.e., the case of a linear autonomous differential equation. In this situation, the splitting of $P E$ is determined by the Jordan canonical form of the matrix for the differential equation. A connected Morse set is an invariant subspace corresponding to Jordan blocks having eigenvalues with consecutive real parts (see Example 1 in §11).

THEOREM 9.2. Let $f$ be chain recurrent on a connected space $Q$. Suppose $\left\{S_{1}, \cdots, S_{k}\right\}$ is a Morse decomposition of $P F$. Then there exist subbundles, $\left\{P E_{1}, \cdots, P E_{k}\right\}$, of $P E$ so that $S_{i}=P E_{i}, i=1, \cdots, k$; and $P E$ is the direct sum of $P E_{1}, \cdots, P E_{k}$.

This theorem and the previous lemmas immediately give:

Corollary 9.3. Let $f$ be a flow on $M$ and $Q$ be a component of the chain recurrent set of $f$. Then each Morse decomposition of $P F$ gives a splitting of $\left.P E\right|_{Q}$ into a direct sum of invariant subbundles.

THEOREM 9.4. If $S$ is an attractor (repeller) for $P F$ and if $c \in \Omega(b, f)$ (respectively, $\left.f^{*}\right)$, then $\operatorname{dim}\left(S \cap P_{c}^{n}\right) \geqslant \operatorname{dim}\left(S \cap P_{b}^{n}\right)$.

The lemma crucial to the proofs of Theorem 9.2 and Theorem 9.4 says that if $b$ and $c$ are points of $M$ and if the $\Omega$-limit set of $b$ contains $c$, then the 
$\Omega$-limit set of an $r$-dimensional subspace in the fiber over $b$ contains at least an $r$-dimensional subspace in the fiber over $c$.

Lemma 9.5. Suppose $b, c \in M$ and $c \in \Omega(b, f)$. For each r-dimensional subspace $P_{b}^{r}$ contained in $P_{b}^{n}$, there exists an $r$-dimensional subspace $P_{c}^{r}$ such that $P_{c}^{r} \subset \Omega\left(P_{b}^{r}, P F\right) \cap P_{c}^{n}$.

Proof. Define $\Omega^{\prime}\left(P_{b}^{r}, \epsilon, t, P F\right) \equiv\{y \mid$ there is an $(\epsilon, t, P F)$-chain from $x$ to $y$ for some $x \in P_{b}^{\eta}$. Proposition 8.2 implies

$$
\Omega\left(P_{b}^{r}, P F\right)=\bigcap_{\epsilon, t>0} \Omega^{\prime}\left(P_{b}^{r}, \epsilon, t, P F\right) .
$$

For each $\epsilon, t>0$ we will show $\Omega^{\prime}\left(P_{b}^{r}, \epsilon, t, P F\right)$ meets $P_{c}^{n}$ in at least an $r$-dimensional subspace. Given $\epsilon$ and a $P_{x}^{r}$ in the fiber over some $x \in M$, there exists a $\delta$ (independent of $x$ ) so that for each $y$ within $\delta$ of $x$ there is a $P_{y}^{r}$ within $\epsilon$ of $P_{x}^{r}$ (in the Hausdorff metric). Obtain this $\delta$ as follows: Take a finite cover of $M$ by bundle charts. Because of local triviality, a $\delta$ can be found for each set in the cover. The desired $\delta$ is the minimum of these and the Lebesgue number of the cover.

Since $c \in \Omega(b, f)$, there is a $(\delta, t, f)$-chain from $b$ to $c$. The preceding implies the existence of an $(\epsilon, t, P F)$-chain from points of $P_{b}^{r}$ to each point of some $P_{c}^{r}$, denoted $P_{c}^{r}(\epsilon, t)$.

Since $\mathrm{Cl} \Omega^{\prime}\left(P_{b}^{r}, \epsilon / 2, t, P F\right) \subset \Omega^{\prime}\left(P_{b}^{r}, \epsilon, t, P F\right), \Omega\left(P_{b}^{r}, P F\right)$ can be expressed as the intersection of a nested family of closed sets. Let $P_{c}^{r}$ be a limit point of the family $P_{c}^{r}(\epsilon, t)$ as $\epsilon \rightarrow 0$ and $t \rightarrow \infty$. Then $P_{c}^{r} \subset \Omega\left(P_{b}^{r}, P F\right)$.

Proof of Theorem 9.2. We use induction on $k$. For $k=1, S_{1}$ must equal $P E$ by a previous remark. So we assume the theorem is true for $k-1$ and prove it for $k$.

Let $\left\{S_{1}, \cdots, S_{k}\right\}$ be a Morse decomposition of $P F$. If $S_{i}=\varnothing$ for any $i, 1 \leqslant i \leqslant k$, delete it and then we are done by induction. Otherwise $S_{1} \neq \varnothing$ and is an attractor. Let $S_{1}^{*}$ denote its dual repeller. Since $S_{i}, i=1, \cdots, k$, are disjoint invariant sets, $S_{i}$ is contained in $S_{1}^{*}$ for $i>1$. We will show the following:

(1) The theorem is true for $\left\{S_{1}, S_{1}^{*}\right\}$.

(2) $\left\{S_{2}, \cdots, S_{k}\right\}$ is a Morse decomposition of $\left.P F\right|_{s_{1}^{*}}$ where, in light of (1), $S_{1}^{*}$ is a bundle over $Q$.

Then induction will apply to the $S_{1}^{*}$ bundle to complete the proof.

(2) is a consequence of Lemma 8.10.

We prove (1) as follows: For ease of notation replace $S_{1}$ by $S$. Remember that $S=\Omega(S, P F)$. Take $b \in Q$ such that $S \cap P_{b}^{n} \neq \varnothing$; by Lemma 9.1, $S \cap P_{b}^{n}=P_{b}^{r}$ for some $r \geqslant 0$. For each $c \in Q$, Proposition 8.5 implies that 
$c \in \Omega(b, f)$. Thus Lemma 9.5 implies that $S \cap P_{c}^{n}$ contains some $P_{c}^{r}$. Interchanging the roles of $b$ and $c$, we see that $S$ meets all fibers in precisely an $r$-dimensional subspace for some $r \geqslant 0$.

Likewise, $S^{*}$ meets all fibers in a $k$-dimensional subspace for some $k$. We show that $r+k=n-1$ : Fix $b \in Q$ and consider a subspace of $P_{b}^{n}$ complementary to $S^{*} \cap P_{b}^{n}=P_{b}^{k}$. Denote this subspace by $P_{b}^{n-k-1}$. By the definition of $S^{*}, \omega\left(P_{b}^{n-k-1}, P F\right)$ is a subset of $S$. Since $S$ is an attractor, $\Omega\left(P_{b}^{n-k-1}, P F\right)$ is also a subset of $S$. But, for each $c \in Q$, Lemma 9.5 implies that $\Omega\left(P_{b}^{n-k-1}, P F\right)$ meets $P_{c}^{n}$ in a subspace of dimension at least $n-$ $k-1$. Therefore, $r=n-k-1$.

To complete the proof we need only show that $S$ (likewise $S^{*}$ ) is a subbundle. If $S^{\prime}$ denotes the pull-back of $S$ to $E$, then $S^{\prime}$ is a closed set of $r+1$-planes in $E$. But Proposition 1.1 applies to $S^{\prime}$ showing that $S^{\prime}$ is a subbundle of $E$.

Definition. A finest Morse decomposition of $P F$ is a Morse decomposition of $P F,\left\{S_{1}, \cdots, S_{k}\right\}$ where $S_{i} \neq \varnothing, i=1, \cdots, k$, so that for each Morse decomposition of $P F,\left\{C_{1}, \cdots, C_{s}\right\}$ where $C_{j} \neq \varnothing, j=1, \cdots, s$ :

(1) $s \leqslant k$, and

(2) for each $S_{i}, i=1, \cdots, k$, there is a $C_{j}, 1 \leqslant j \leqslant s$, so that $S_{i} \subset C_{j}$.

REMARK. A finest Morse decomposition of $P F$ is unique up to a reordering of the Morse sets.

Lemma 9.6. Let $\left\{S_{1}, \cdots, S_{k}\right\}$ and $\left\{T_{1}, \cdots, T_{s}\right\}$ be two Morse decompositions of $P F$. Define sets $C_{s(i-1)+j} \equiv S_{i} \cap T_{j}$ for $i=1, \cdots, k$ and $j=$ $1, \cdots, s$. Then $\left\{C_{1}, \cdots, C_{s k}\right\}$ is a Morse decomposition of $P F$.

REMARK. $\left\{C_{1}, \cdots, C_{s k}\right\}$ is called the intersection of $\left\{S_{1}, \cdots, S_{k}\right\}$ and $\left\{T_{1}, \cdots, T_{s}\right\} .\left\{S_{1}, \cdots, S_{k}\right\} \cap\left\{T_{1}, \cdots, T_{s}\right\}$ is the same as $\left\{T_{1}, \cdots, T_{s}\right\} \cap$ $\left\{S_{1}, \cdots, S_{k}\right\}$ after reordering.

THEOREM 9.7. Let $f$ be a chain recurrent on a connected space $Q$. Then there is a finest Morse decomposition of PF over $Q$. (For uniqueness see Corollary 11.7.)

Proof. Fix $b \in Q$. Since $D\left(P_{b}^{n}\right)$ is finite (Theorem 7.4), there are only a finite number of sequences of subspaces of $P_{b}^{n},\left\{K_{1}, \cdots, K_{m}\right\}$, so that $K_{i}=$ $T_{i} \cap P_{b}^{n}$ where $\left\{T_{1}, \cdots, T_{m}\right\}$ is a Morse decomposition of $P F$. For each such sequence take a Morse decomposition which meets $P_{b}^{n}$ in that sequence. Let $\left\{S_{1}, \cdots, S_{r}\right\}$ be the intersection of all these Morse decompositions, after deleting any empty intersections. For each Morse decomposition $\left\{T_{1}, \cdots, T_{m}\right\}$ where $T_{j} \neq \varnothing, j=1, \cdots, m$, this construction and Theorem 9.2 imply that $m \leqslant r$ 
and that for each $S_{i}$ there is a $T_{j}$ so that $S_{i} \cap P_{b}^{n} \subset T_{j} \cap P_{b}^{n}$. In fact, for each $c \in Q$ and $S_{i}$, there is a $T_{j}$ where $j$ depends on $c$ so that $S_{i} \cap P_{c}^{n} \subset$ $T_{j} \cap P_{c}^{n}$ : If there is a Morse decomposition $\left\{T_{1}, \cdots, T_{m}\right\}$ so that $S_{i} \cap P_{c}^{n} \not \subset$ $T_{j} \cap P_{c}^{n}$ for any $j$, then $\left\{S_{1}, \cdots, S_{r}\right\} \cap\left\{T_{1}, \cdots, T_{m}\right\}$ is a Morse decomposition having more than $r$ nonempty Morse sets. But this contradicts the maximality of $r$.

Given $\left\{T_{1}, \cdots, T_{m}\right\}$, we associate to each $c \in Q$ a sequence of $r$ integers, $j_{i}(c), i=1, \cdots, r$, where $j_{i}(c)$ is the integer such that $S_{i} \cap P_{c}^{n} \subset T_{j_{i}(c)}$ $\cap P_{c}^{n}$. To show $\left\{S_{1}, \cdots, S_{r}\right\}$ is the finest Morse decomposition, it suffices to show $j_{i}(c)$ is constant as a function of $c$ for $i=1, \cdots, r$. Let $H \equiv\{c \in Q \mid$ $j_{i}(c)=j_{i}(b)$ for $\left.i=1, \cdots, r\right\} . H \neq \varnothing$ and $H$ is both open and closed because the $S_{i}$ 's and $T_{j}$ 's are subbundles so their intersections with the fiber are continuous functions of the base. Thus $H=Q$ since $Q$ is connected.

As a consequence of Theorem 9.7, all Morse sets over a connected set on which $f$ is chain recurrent are linear spans of the Morse sets in the finest Morse decomposition. Thus quasi-Morse sets are Morse sets. Since, in general, components of $R(P F)$ are quasi-Morse sets, the components of $R(P F)$ over a connected, chain recurrent set are precisely the finest Morse sets.

In the case of the linear autonomous differential equation, each Morse set in the finest Morse decomposition is the span of the Jordan blocks having eigenvalues with the same real part. And, as mentioned earlier, every connected Morse set is the span of all Jordan blocks having eigenvalues with consecutive real parts.

EXAMPLE. Strict inequality in Theorem 9.4 occurs often. In fact, for any Morse set $S$, the dimension of $S \cap P_{b}^{n}$ is only upper semicontinuous as a function of $b$ (since $S$ is closed and meets fibers in linear subspaces). Also, if $Q$ is only a Morse set of $f$ there may not be a splitting over $Q$ as in Theorem 9.2. This example illustrates both these statements.

Consider a smooth flow $f$ on the circle $S^{1}$. Let $[b, c]$, an arc joining the rest points $b$ and $c$, be a repeller and some other point in $S^{1}-[b, c]$ be an attractor. The flow in $[b, c]$ goes from $b$ to $c$ (Figure 1 ). $[b, c]$ is a Morse set of $f$. Parameterize the arc $(b, c)$ by $R$. Take an $R^{2}$-bundle over $S^{1}$ with the flow $F$ over $[b, c]$ being the solutions of the following differential equations: Over $b$, use the system $\dot{x}=-x$ and $\dot{y}=y$. Over $c$, use the system $\dot{x}=x$ and $\dot{y}=y$. Over $(b, c)$, use the system $\dot{x}=(2 / \pi)(\arctan t) x$ and $\dot{y}=y$ for $t \in R$. $F$ induces $P F$ on a $P^{1}$-bundle over $[b, c]$ given in Figure 2. Let $x$ denote the repeller over $b$, and $y$ the attractor. Let $z$ be the $\omega$-limit set of the orbit over $(b, c)$ with $\alpha$-limit set $y$. A Morse decomposition over $[b, c]$ has $x$ as the repeller and the orbit from $y$ to $z$ plus the fiber over $c$ as the attractor. So the attractor increases fiber dimension at 
$c$ and neither attractor nor repeller are subbundles over $[b, c]$.

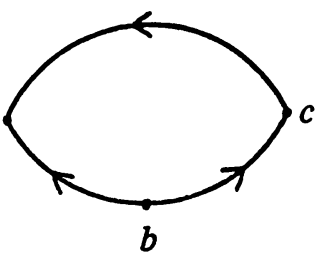

Figure 1

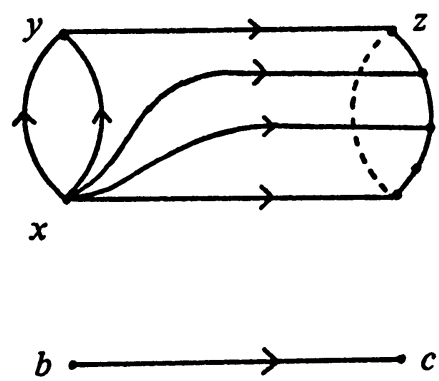

FIGURE 2

Theorem 9.4 is just the first step in tying the Morse structure of the flow in $P E$ to that of the flow in $M$. In the preceding example, $b$ and $c$ are components of the chain recurrent set and Morse sets in a Morse decomposition, and $c \in \Omega(b, f)$. Notice that the attractor in the Morse decomposition of $P F$ increases fiber dimension as the flow proceeds from $b$ to $c$ and the repeller loses fiber dimension. This is the force of Theorem 9.4, i. e., as the flow moves down through the filtration of $f$ (down in terms of the indexing in a Morse filtration) the attractors in a Morse decomposition of $P F$ cannot decrease fiber dimension but may increase fiber dimension and the repellers cannot increase but may decrease; and an attractor increase must be balanced by a repeller decrease. Theorem 9.4 guarantees this, provided the Morse sets of the decomposition of $f$ are exactly components of $R(f)$.

To give a more complete picture of $P F$ we need to study how the finest Morse decomposition changes from over one component of $R(f)$ to over the next component, i. e., how does the splitting over one component of $R(f)$ link up with the splitting over another component.

10. Hyperbolicity. As in previous sections, let $(F, f)$ be a linear flow on a vector bundle $(E, M, \pi, M)$. For $x \in E$, let $|x|$ denote $g(x, x)^{1 / 2}$ where $g$ is a Riemannian metric on $E$.

Definition. $(F, f)$ is hyperbolic on $E$ if

(1) there exists a splitting of $E$ into the direct sum of two invariant subbundles, $E^{s}$ and $E^{u}$; and

(2) there are real numbers $\alpha>0$ and $0<\lambda<1$ so that, for $t \geqslant 0$, $\left|F_{t}(x)\right| \leqslant \alpha \lambda^{t}|x|$ for $x \in E^{s}$ and $\left|F_{t}^{*}(x)\right| \leqslant \alpha \lambda^{t}|x|$ for $x \in E^{u}$.

Remark. Suppose $f$ is $C^{r}$ on a compact $C^{r}$-manifold $M$ and $F_{t} \equiv$ $T f_{t}$. Let $X: M \rightarrow T M$ be the vector field for $f$. If $f$ is hyperbolic in the usual sense [20], then $(F, f)$ is hyperbolic according to our definition on $T M / X$, the quotient bundle obtained from $T M$ by factoring out the tangent direction to $f$. 
In order to prove the equivalence between hyperbolicity and the zero section $Z$ of $E$ being an isolated invariant set, we need the following observations. Lemma 10.1 can be found in [2].

LEMMA 10.1. Let $I$ be an isolated invariant set and $N$ a compact neighborhood of I. Suppose that for each $x \in N-I$, there exists $t<0 \quad(t>0)$ such that $x \cdot t \notin N$. Then $I$ is an attractor (respectively, a repeller). ( $N$ will be called an attractor neighborhood for $I$.)

REMARK. Also, because of the linearity of $F, Z$ is isolated if and only if $F$ has no nonzero bounded orbits (bounded in terms of $g$ ).

THEOREM 10.2. Suppose $f$ is chain recurrent on a connected space $M$. Then $(F, f)$ is hyperbolic on $E$ if and only if $Z$ is an isolated invariant set.

Proof. If $(F, f)$ is hyperbolic, then $F$ has no nonzero bounded orbits. Thus $Z$ is isolated.

An outline of the proof of sufficiency is: Use the two asymptotic sets to $Z$ to define sets in the bundle $P E$. These sets are the attractor and repeller in a Morse decomposition of $P F$. Therefore, by Theorem 9.2, $P E$ splits into the direct sum of two subbundles. We pull these subbundles back to $E$ and show there is exponential growth in the attractor and decay in the repeller.

Define asymptotic sets as follows:

$A+\equiv\left\{x \in E \mid\right.$ there exists an $a \in R$ so that $|y|<a$ for all $\left.y \in x \cdot R^{+}\right\}$ and

$A-\equiv\{x \in E \mid$ there exists an $a \in R$ so that $|y|<a$ for all $y \in x \cdot R\}$.

Given $x \in A+$ and a neighborhood of $Z$, some scalar multiple of $x$ is in this neighborhood for all $t \geqslant 0$ and, thus, goes to 0 as $t \rightarrow \infty$. By linearity, $x$ goes to 0 as $t \rightarrow \infty$. Likewise for $x \in A-, x$ goes to 0 as $t \rightarrow-\infty$. $A^{-}$ and $A+$ meet each fiber of $E$ in a linear subspace, possibly $\{0\}$. Since $Z$ is isolated, $A+\cap A^{-}=Z$. We may assume neither $A^{-}$nor $A+$ equals $E$. This implies, via Lemma 10.1, that neither equals $Z$. Upon identification, $\left(A^{-}\right)-Z$ and $(A+)-Z$ give the disjoint, nonempty sets $S_{1}$ and $S_{2}$ in $P E$.

Take an isolating block $B$ for $Z$. There is an $r>0$ so that Int $B$ contains the disk bundle $D(r)$ of radius $r$, i.e., $D(r) \equiv\{x \in E|| x \mid \leqslant r\}$. If $x \in$ $A+\cap D(r)$, then the line segment from 0 to $x$ is in Int $B$, and, since $x$ goes to 0 as $t \rightarrow \infty$, Lemma 5.1 implies that $x \cdot R^{+} \subset$ Int $B$. So points of $A+\cap D(r)$ stay in $B$ for all $t \geqslant 0$ and, likewise, points of $A-\cap D(r)$ stay in $B$ for all $t \leqslant 0$. Let $S(r)$ denote the sphere bundle of radius $r$. Since $A+$ and $A-$ are linear, $A+\cap S(r)$ and $A-\cap S(r)$ give $S_{2}$ and $S_{1}$ after identification. 
We claim that $S_{2}$ is a repeller: Because $A \pm \cap S(r)$ are closed, there are disjoint open sets $U$ and $V$ in $P E$ so that $S_{2} \subset U$ and $S_{1} \subset V$. Pull $U$ and $V$ back to disjoint neighborhoods, $U^{\prime}$ and $V^{\prime}$, of $A+$ and $A-$. It is a general fact ([2], [7], [9]) that points of $B$ near those which remain in $B$ for all $t \geqslant 0$ leave $B$ near points which remain in $B$ for all $t \leqslant 0$. Because of this and the compactness of $A+\cap S(r)$, there is a closed neighborhood $N^{\prime}$ of $A+\cap S(r)$ in $U^{\prime}$ such that for each $x \in N^{\prime}-(A+\cap S(r))$ there is a $t>0$ so that $x \cdot t \in V^{\prime}$. Thus $N^{\prime}$, after identification, gives a repeller neighborhood for $S_{2}$ in $P E$. By Lemma 10.1, $S_{2}$ is a repeller.

Likewise, $S_{1}$ is an attractor.

So $S_{1}$ and $S_{2}$ are Morse sets and, by Lemma 8.9 and Theorem 9.2, each meets every fiber in a subspace of constant dimension. Clearly, $S_{2} \subset S_{1}^{*}$. If $S_{1}^{*}-S_{2} \neq \varnothing$, then choose $x$ in a Morse set contained in $S_{1}^{*}-S_{2}$. The closure of the orbit of $x$ and $S_{2}$ are separated by open sets $U$ and $V$. Let $y \in S_{1}$ and be in the fiber containing $x$. Consider the $P^{1}$ spanned by $x$ and $y$. Since $S_{1}$ is an attractor, $y$ must be a negative expansion point so all points of this $P^{1}$ near $y$ go to $x$ as $t \rightarrow-\infty$, i.e., there is a $T<0$ so that, for all $t \leqslant T$ and $z \neq y \in P^{1}, z \cdot t$ is in the open set $U$ containing the orbit of $x$. But, as above, the points of $B$ near points which remain in $B$ for all $t \leqslant 0$ leave $B$, in large negative time, near points which remain in $B$ for all $t \geqslant 0$. So there are points in $P^{1}$ near $y$ which go into $V$, the neighborhood of $S_{2}$, for arbitrarily large negative time. This is a contradiction. Thus $S_{2}=S_{1}^{*}$ and $\left\{S_{1}, S_{2}\right\}$ is a Morse decomposition of $P F$. Theorem 9.2 implies that $P E$ splits into the direct sum of subbundles $S_{1}$ and $S_{2}$. So $E$ splits into the direct sum of $A^{-}$and $A+$.

We claim that $\left\{F_{t}\right\}$ decays exponentially in $A+$. Consider the unit sphere bundle $S(1)$ in $E$. Take the sphere bundle of radius $1 / 2, S(1 / 2)$, and a block $B$ inside $S(1 / 2)$, i.e., $B \subset$ Int $D(1 / 2)$. For some $r>0, S(r)$ is contained in Int $B$. For each $x \in A+\cap S(1)$, there is $T_{x}>0$ so that $x \cdot T_{x}$ is inside $S(r)$. By continuity, take a neighborhood $U_{x}$ of $x$ in $A+\cap S(1)$ so that $U_{x} \cdot T_{x}$ is inside $S(r)$. By Lemma 5.1, $U_{x} \cdot\left[T_{x}, \infty\right) \subset$ Int $B$. Take a finite subcover of $A+\cap S(1)$ by these $U_{x}$ 's and let $T_{1}$ be the maximum of the corresponding $T_{x}$ 's. So for each $x \in A+\cap S(1),\left|F_{t}(x)\right| \leqslant 1 / 2=1 / 2|x|$ for all $t \geqslant T_{1}$. By linearity, for each $x \in A+,\left|F_{t}(x)\right| \leqslant 1 / 2|x|$ for all $t \geqslant T_{1}$. Let $\lambda \equiv(1 / 2)^{1 / T_{1}}$. For each $x \in A+,\left|F_{t}(x)\right| \leqslant 2 \lambda^{t}|x|$ for all $t \geqslant T_{1}$ : For $t \geqslant T_{1}$, there is a positive integer $k$ and a real number $s, 0<s<T_{1}$, so that $t=k T_{1}+s$. Then

$$
\left|F_{t}(x)\right|=\left|F_{T_{1}}\left(F_{(k-1) T_{1}+s}(x)\right)\right| \leqslant 1 / 2\left|F_{(k-1) T_{1}+s}(x)\right| \leqslant \cdots \leqslant(1 / 2)^{k}|x| \leqslant 2 \lambda^{t}|x| .
$$

By compactness of $\left[0, T_{1}\right]$ and linearity, we choose $\alpha \in R^{+}$so that $\left|F_{t}(x)\right| \leqslant \alpha \lambda^{t}|x|$ for all $x \in A+$ and all $t \geqslant 0$. 
Similarly, $\left\{F_{t}^{*}\right\}$ decays exponentially on $A-$. So $(F, f)$ is hyperbolic.

REMARK. The equivalence in Theorem 10.2 depends on the condition that $f$ is chain recurrent on $M$. Consider the example of $\S 9$. The flow on the $R^{2}$. bundle over $[b, c]$ is not hyperbolic but the zero section is isolated since orbits become unbounded as they approach the fiber over $c$.

11. Growth rates for orbits in a Morse decomposition. Now we will investigate the different exponential growth rates in the pull-backs of Morse sets of a Morse decomposition over a connected subset $Q$ of $M$ on which $f$ is chain recurrent.

LEMMA 11.1. Suppose $E^{\prime}$ is an invariant subbundle of $E$ over $M$. If for each $x \in E^{\prime}$ there is $t_{k} \rightarrow \infty$ so that $\left|F_{t_{k}}(x)\right| \rightarrow \infty$, then $Z$ over $M$ is a repeller for $\left.F\right|_{E^{\prime}}$.

Proof. Any disk bundle in $E^{\prime}$ is a repeller neighborhood for $Z$ so Lemma 10.1 gives the result.

LEMMA 11.2. Suppose $E^{\prime}$ is an invariant subbundle of $E$. If for each $x \in E^{\prime}$ there is a $t_{k} \rightarrow \infty$ so that $\left|F_{t_{k}}(x)\right| \rightarrow 0$, then $Z$ is an attractor for $\left.F\right|_{E^{\prime}}$

Proof. We claim that the unit disk bundle $D(1)$ is an attractor neighborhood for $Z$ :

There is a $T>0$ so that, for each $x \in S(1)$, there is a $t \in[0, T]$ such that $|x \cdot t| \leqslant 1 / 2|x|$. If not, for each $i>0$ there is $x_{i} \in S(1)$ so that $\left|x_{i} \cdot t\right|$ $>1 / 2$ for all $t \in[0, i]$. Without loss of generality, $x_{i} \rightarrow x \in S(1)$. Since $\left|x \cdot t_{k}\right| \rightarrow 0$ as $t_{k} \rightarrow \infty$, there is an $N$ so that for all $t_{k} \geqslant N,\left|x \cdot t_{k}\right|<1 / 2$. Consider $t_{1}$ and $\left|x \cdot t_{1}\right|<1 / 2$. For $i$ large, $\left|x_{i} \cdot t_{1}\right|<1 / 2$. So for some $i>t_{1}$, $\left|x_{i} \cdot t_{1}\right|<1 / 2$, which contradicts $\left|x_{i} \cdot t\right|>1 / 2$ for all $t \in[0, i]$. Also, by the linearity of $F$, for each $x \in D(1)$ we have $|x \cdot t|<1 / 2|x|$ for some $t \in[0, T]$.

Suppose there is $y \neq 0 \in D(1)$ such that $y \cdot(-\infty, 0] \subset D(1)$. Let $y \cdot s$, for $0 \leqslant s \leqslant T$, be such that $|y \cdot s|=\inf \{|y \cdot t| \mid t \in[0, T]\}$. Pick $i \in R$ so that $2^{i}<|y \cdot s|$. Let $z \equiv y \cdot(-i T)$. Then $|z| \leqslant 1$, and there is a $t \in[0, T]$ so that $|z \cdot t| \leqslant 1 / 2|z|$. Let $z^{\prime}=z \cdot t$; then there is a $t^{\prime} \in[0, T]$ so that $\left|z^{\prime} \cdot t^{\prime}\right| \leqslant 1 / 2\left|z^{\prime}\right| \leqslant 1 / 4|z| \leqslant 1 / 4$. Continue this process until we have $\widetilde{z} \in y \cdot[0, T]$ and $|\tilde{z}| \leqslant 1 / 2^{i}<|y \cdot s|$. This is a contradiction. Thus $y \cdot(-\infty, 0] \not \subset D(1)$ and $D(1)$ is an attractor neighborhood.

LEMMA 11.3. Let $\left\{S_{1}, \cdots, S_{k}\right\}$ be a Morse decomposition of PF over $Q$ with $\left\{E_{1}, \cdots, E_{k}\right\}$ being the corresponding pull-backs to $E$. Then at most one $E_{i}$ contains two points $x, y \neq 0$ so that $\left|F_{t}(x)\right|+0$ as $t \rightarrow \infty$ and $\left|F_{t}(y)\right|+\infty$ as $t \rightarrow \infty$. 
Proof. Suppose $E_{i}$ and $E_{j}$ both contain such points, $x_{i}, y_{i} \in E_{i}$ and $x_{j}, y_{j} \in E_{j}$. Without loss of generality assume $S_{i}$ is an attractor and $S_{j}$ is a repeller. Thus, points of a $P^{1}$ determined by a point of $S_{i}$ and a point of $S_{j}$ go to $S_{i}$ as $t \rightarrow \infty$. There is a sequence $t_{k} \rightarrow \infty$ so that $\left|y_{i} \cdot t_{k}\right|$ is bounded. For each $z \in S_{j}$ and $w \neq z$ in the $P^{1}$ determined by $z$ and $y_{i}, \rho\left(w \cdot t, y_{i} \cdot t\right)$ $\rightarrow 0$ as $t \rightarrow \infty$. Thus $\left|z \cdot t_{k}\right| \rightarrow 0$ as $t_{k} \rightarrow \infty$. Lemma 11.2 implies that $Z$ is an attractor for $\left.F\right|_{E_{j}}$. Then Theorem 10.2 implies that $|z \cdot t| \rightarrow 0$ as $t \rightarrow \infty$ for all $z \in E_{j}$. This contradicts the fact that $\left|x_{j} \cdot t_{m}\right| \nrightarrow 0$ for a sequence $t_{m} \rightarrow \infty$.

Corollary 11.4. Let $\left\{S_{1}, \cdots, S_{k}\right\}$ be a Morse decomposition of PF over $Q$ with $\left\{E_{1}, \cdots, E_{k}\right\}$ being the corresponding pull-backs to $E$. Then there is at most one $E_{i}$ so that $Z$ is not an isolated invariant set of $\left.F\right|_{E_{i}}$.

PRoof. If $Z$ is not an isolated invariant set of $\left.F\right|_{E_{i}}$, then there is some $x \in E_{i}(\neq 0)$ so that $\left|F_{t}(x)\right|+0$ as $t \rightarrow \infty$ by Lemma 11.2 and some $y \in$ $E_{i}$ so that $\left|F_{t}(y)\right| \nrightarrow \infty$ by Lemma 11.1. Lemma 11.3 gives the result.

LEMMA 11.5. Let $E^{\prime}$ be a subbundle of $E$ over $M$. Then there exists $\alpha, \beta, \gamma, \delta \in R^{+}$where $\gamma \leqslant 1$ and $\delta \geqslant 1$ so that $\beta \gamma^{t}|x| \leqslant\left|F_{t}(x)\right| \leqslant \alpha \delta^{t}|x|$ for all $x \in E^{\prime}$ and $t \geqslant 0$.

Proof. Let $S(1)$ denote the unit sphere bundle in $E^{\prime}$. Let $\gamma \equiv$ $\inf \{|x \cdot t| \mid x \in S(1)$ and $t \in[0,1]\}$. So $0<\gamma \leqslant 1$. By linearity, $\gamma|x| \leqslant$ $|x \cdot t|$ for all $x \in E^{\prime}$ and $t \in[0,1]$. For each $t>1, t=k+s$ where $k$ is a positive integer and $0<s<1$. So, for $x \in E^{\prime}$,

$$
|x \cdot t| \geqslant \gamma^{k}|x \cdot s| \geqslant \gamma^{k+1}|x| \geqslant \gamma^{t+1}|x| \text {. }
$$

Therefore, $|x \cdot t| \geqslant \beta \gamma^{t}|x|$ for all $x \in E^{\prime}$ and $t \geqslant 0$.

Let $\delta \equiv \sup \{|x \cdot t| \mid x \in S(1)$ and $t \in[0,1]\}$. Then $1 \leqslant \delta<\infty$. By linearity, $|x \cdot t| \leqslant \delta|x|$ for all $x \in E^{\prime}$ and $t \in[0,1]$. As above, for $t>1$, $t=k+s$. And $|x \cdot t| \leqslant \delta^{k}|x \cdot s| \leqslant \delta^{t+1}|x|$ for $x \in E^{\prime}$. Thus, $|x \cdot t| \leqslant$ $\alpha \delta^{t}|x|$ for all $x \in E^{\prime}$ and $t \geqslant 0$.

DEFinition. Let $E^{\prime}$ be an invariant subbundle over $M$. Define $\lambda \equiv$ $\sup \left\{\lambda \in R|| e^{-\lambda t} F_{t}(x) \mid \rightarrow \infty\right.$ as $t \rightarrow \infty$ for all $\left.x \neq 0 \in E^{\prime}\right\}$, where $e^{-\lambda t} F_{t}(x)$ denotes the vector whose components are $e^{-\lambda t}$ times each component of $F_{t}(x)$. And define $\bar{\lambda} \equiv \inf \left\{\lambda \in R|| e^{-\lambda t} F_{t}(x) \mid \rightarrow 0\right.$ as $t \rightarrow \infty$ for all $\left.x \neq 0 \in E^{\prime}\right\}$.

Remarks. Lemma 11.5 implies that $\lambda$ and $\bar{\lambda}$ are finite. And, for $x \in$ $E^{\prime}$, if $\left|e^{-\lambda t} F_{t}(x)\right| \rightarrow r \neq 0$ as $t \rightarrow \infty$, then $\lambda \in[\underline{\lambda}, \bar{\lambda}]$. The closed interval $[\lambda, \bar{\lambda}]$ is called the (positive) growth rate interval of $F$ on $E^{\prime}$. The growth rate interval of $F^{*}$ is the negative growth rate interval of $F$. 
LEMMA 11.6. Let $S$ be a Morse set over $Q$ with pull-back $E^{\prime}$ and let $\lambda_{t} \rightarrow \lambda$ as $i \rightarrow \infty$. If $\left|e^{-\lambda t} F_{t}(x)\right| \rightarrow \infty$ as $t \rightarrow \infty$ for all $x \in E^{\prime}$, then for large $i,\left|e^{-\lambda i t} F_{t}(x)\right| \rightarrow \infty$ as $t \rightarrow \infty$ for all $x \in E^{\prime}$. Also, if $\left|e^{-\lambda t} F_{t}(x)\right|$ $\rightarrow 0$ as $t \rightarrow \infty$ for all $x \in E^{\prime}$, then for large $i,\left|e^{-\lambda_{i} t} F_{t}(x)\right| \rightarrow 0$ as $t \rightarrow$ $\infty$ for all $x \in E^{\prime}$.

Proof. For the first assertion, let $G_{t}(x) \equiv e^{-\lambda t} F_{t}(x)$ for all $x \in E^{\prime}$ and $t \in R$. Then $\left\{G_{t}\right\}$ is a flow on $E^{\prime}$ and Lemma 11.1 implies that $Z$ is a repeller for $G$. Theorem 10.2 implies that $G$ grows exponentially for $t>0$, so for some $T>0, G_{T}$ takes the sphere bundle $S(1)$ into Ext $D(1)$. For large $i,\left\{e^{-\lambda_{i} t} F_{t}\right\}$ is close to $\left\{G_{t}\right\}$ in the compact open topology so $e^{-\lambda_{i} T} F_{T}$ takes $S(1)$ into Ext $D(1)$. By linearity, $D(1)$ is a repeller neighborhood for $\left\{e^{-\lambda_{i} t} F_{t}\right\}$ for large $i$. Theorem 10.2 implies that $\left|e^{-\lambda_{i} t} F_{t}(x)\right| \rightarrow \infty$ as $t \rightarrow$ $\infty$ for all $x \in E^{\prime}$.

The second assertion follows from Lemma 11.2 and Theorem 10.2.

THEOREM 11.7. Let $\left\{S_{1}, \cdots, S_{k}\right\}$ be a Morse decomposition of PF over $Q$ with pull-backs $\left\{E_{1}, \cdots, E_{k}\right\}$. Let $\left[\underline{\lambda}_{i}, \bar{\lambda}_{i}\right]$ denote the growth rate interval for $E_{i}, i=1, \cdots, k$. Then $\left[\underline{\lambda}_{i}, \bar{\lambda}_{i}\right] \cap\left[\underline{\lambda}_{j}, \bar{\lambda}_{j}\right]=\varnothing$ for $i \neq j$.

Proof. Suppose $\lambda \in\left[\underline{\lambda}_{i}, \bar{\lambda}_{i}\right] \cap\left[\underline{\lambda}_{j}, \bar{\lambda}_{j}\right]$ for $i \neq j$. Lemma 11.6 implies that there are points $x_{i}, y_{i} \neq 0 \in E_{i}$ and $x_{j}, y_{j} \neq 0 \in E_{j}$ so that $\left|e^{-\lambda t} F_{t}\left(x_{m}\right)\right|$ $\rightarrow 0$ as $t \rightarrow \infty$ and $\left|e^{-\lambda t} F_{t}\left(y_{m}\right)\right|+\infty$ as $t \rightarrow \infty, m=i, j$. Define $G_{t}(z)$ $=e^{-\lambda t} F_{t}(z)$ for all $z \in E$. $G$ is a flow on $E$ and $P G=P F$ on $\dot{P E}$. Thus $\left\{S_{1}, \cdots, S_{k}\right\}$ is a Morse decomposition for $P G$ with pull-backs $\left\{E_{1}, \cdots, E_{k}\right\}$. The existence of $x_{i}, y_{i} \in E_{i}$ and $x_{j}, y_{j} \in E_{j}$ contradicts Lemma 11.3.

Corollary 11.8. Let $S$ be a Morse set over $Q$ with pull-back $E^{\prime}$. If $[\underline{\lambda}, \bar{\lambda}]$ is the growth rate interval of $F$ on $E^{\prime}$, then $[-\bar{\lambda},-\underline{\lambda}]$ is the growth rate interval of $F^{*}$ on $E^{\prime}$.

Proof. For each $\lambda>\bar{\lambda}$, consider the flow $G_{t}(x) \equiv e^{-\lambda t} F_{t}(x)$ for $x \in$ $E^{\prime}$. For $x \in E^{\prime},\left|G_{t}(x)\right| \rightarrow 0$ as $t \rightarrow \infty$, so $Z$ is an attractor for $G$ by Lemma 11.2. Theorem 10.2 implies that $\left|G_{t}(x)\right| \rightarrow \infty$ as $t \rightarrow-\infty$ for $x \neq$ $0 \in E^{\prime}$. Let $[\underline{\alpha}, \bar{\alpha}]$ be the positive growth rate interval of $F^{*}$. But $\left|e^{(-\lambda)(-t)} F_{-t}(x)\right| \rightarrow \infty$ as $t \rightarrow \infty$, so $-\lambda \leqslant \underline{\alpha}$. Thus $-\bar{\lambda} \leqslant \underline{\alpha}$. Likewise, for $\lambda<\underline{\lambda}$, we see that $-\lambda \geqslant \bar{\alpha}$. Thus $-\lambda \geqslant \overline{\bar{\alpha}}$.

Applying the preceding argument to the flow $F^{*}$ with growth interval $[\underline{\alpha}, \bar{\alpha}]$ gives $-\bar{\alpha} \leqslant \underline{\lambda}$ and $-\alpha \geqslant \bar{\lambda}$. Therefore $\bar{\alpha}=-\underline{\lambda}$ and $\underline{\alpha}=-\bar{\lambda}$, and so $[\underline{\alpha}, \bar{\alpha}]=[-\bar{\lambda},-\underline{\lambda}]$.

Corollary 11.9. Let $\left\{S_{1}, \cdots, S_{k}\right\}$ be a finest Morse decomposition over 
$Q$ with pull-backs $\left\{E_{1}, \cdots, E_{k}\right\}$. Let $\left[\underline{\lambda}_{i}, \bar{\lambda}_{i}\right]$ denote the growth rate interval for $E_{i}, i=1, \cdots, k$. If $F$ is not hyperbolic on $E$, then there is an $r, 1 \leqslant$ $r \leqslant k$, so that $\bar{\lambda}_{i}<0$ for all $i>r$ and $\underline{\lambda}_{i}>0$ for all $i<r$. Furthermore, $\underline{\lambda}_{i}<\underline{\lambda}_{j}$ if $i>j$.

.Proof. Theorem 10.2 implies that $Z$ is not isolated in some $E_{i}$. Corollary 11.4 implies there is only one such $E_{i}$, say $E_{r}$, and $0 \in\left[\underline{\lambda}_{r}, \bar{\lambda}_{r}\right]$. Also, for $i \neq r$, orbits in $E_{i}$ either all decay exponentially or grow exponentially as $t \rightarrow \infty$ because $\left\{S_{1}, \cdots, S_{k}\right\}$ is a finest Morse decomposition.

For $i>r, S_{r}$ is contained in an attractor and $S_{i}$ is contained in its dual repeller. Thus, points of a $P^{1}$ determined by a point of $S_{r}$ and a point of $S_{i}$ go to $S_{r}$ as $t \rightarrow \infty$. For some $y \in E_{r}$ there is a sequence $t_{k} \rightarrow \infty$ so that $\left|y \cdot t_{k}\right|$ is bounded. Thus, for $z \in E_{i},\left|z \cdot t_{k}\right| \rightarrow 0$ as $t \rightarrow \infty$. So $\bar{\lambda}_{i}<0$.

For $i<r$, a similar argument shows $\underline{\lambda}_{i}>0$.

For $i>j, S_{j}$ is contained in an attractor and $S_{i}$ is contained in its dual repeller. Consider the flow $G_{t} \equiv e^{-\lambda_{j} t} F_{t}$. The preceding argument shows that $\underline{\lambda}_{j} \geqslant \underline{\lambda}_{i}$.

REMARKS. If $F$ is hyperbolic, then the final inequality in Corollary 11.9 holds but no growth rate interval contains 0 . Also, Corollary 11.9 implies that a finest Morse decomposition is unique, i.e., the Morse sets cannot be reordered to give another Morse decomposition.

THEOREM 11.10. Suppose $E$ splits over $Q$ into the direct sum of invariant subbundles $\left\{E_{1}, \cdots, E_{k}\right\}$. Let $\left[\lambda_{i}, \bar{\lambda}_{i}\right]$ denote the growth rate interval for $E_{i}, i=1, \cdots, k$. Let $\left\{S_{1}, \cdots, S_{k}\right\}$ be the corresponding subbundles in PE. If the growth intervals are pairwise disjoint, then $\left\{S_{1}, \cdots, S_{k}\right\}$ is a Morse decomposition of $P F$.

Proof. Use induction on $k$. For $k=1$, the result is trivial. Assume the results for a splitting into $k-1$ subbundles and prove it for $\left\{E_{1}, \cdots, E_{k}\right\}$. Without loss of generality, assume $\underline{\lambda}_{i}>\underline{\lambda}_{j}$ if $j>i$. Let $\lambda$ be a real number such that $\bar{\lambda}_{2}<\lambda<\underline{\lambda}_{1}$. Define the flow $G_{t}(x)=e^{-\lambda t} F_{t}(x)$ for all $x \in E$. Let $E^{\prime}$ be the direct sum of $\left\{E_{2}, \cdots, E_{k}\right\}$. Then $E$ is the direct sum of $E_{1}$ and $E^{\prime}$ both invariant under $G$. The growth rate interval for $E_{1}$ with respect to the flow $G$ is to the right of 0 on the number line. And the growth rate interval for $E^{\prime}$ is to the left of 0 . Thus $Z$ is isolated for the flow $G$ on $E$. According to Theorem $10.2, E^{\prime}=A+$ and $E_{1}=A^{-}$so $E_{1}$ and $E^{\prime}$ give rise to a Morse decomposition $\left\{S_{1}, S\right\}$ of $P G$. But $P G=P F$. By induction, $\left\{S_{2}, \cdots, S_{k}\right\}$ is a Morse decomposition of $\left.P F\right|_{s}$. Therefore, $\left\{S_{1}, S_{2}, \cdots, S_{k}\right\}$ is a Morse decomposition of $P F$. 
EXAmple 1. Let $Q$ be a rest point and so the bundle $E$ over $Q$ is an $n$-dimensional vector space. $F$ over $Q$ is given by $F_{t}(x)=e^{A t} x$ for $x \in E$ where $A$ is an $n \times n$ real matrix. Let $\lambda_{1}, \cdots, \lambda_{k}$ denote the distinct real parts of the eigenvalues of $A$ with $\lambda_{i}>\lambda_{j}$ if $i^{\prime}<j$. For each $i=1, \cdots, k$, define the subbundle $E_{i}$ to be the span of the invariant subspaces corresponding to the Jordan blocks of $A$ with eigenvalues having real part $\lambda_{i}$. Then $E$ is the direct sum of $\left\{E_{1}, \cdots, E_{k}\right\}$. The growth rate interval of $E_{i}$ is $\lambda_{i}, i=1, \cdots, k$. So, by Theorem 11.10, the corresponding subbundles $\left\{S_{1}, \cdots, S_{k}\right\}$ of $P E=$ $P^{n-1}$ give a Morse decomposition of $P F$. Since these growth rate intervals are points, Theorem 11.7 implies that $\left\{S_{1}, \cdots, S_{k}\right\}$ is the finest Morse decomposition of $P F$, and $P F$ is a chain recurrent on each $S_{i}$.

If $Q$ is a periodic orbit, then the situation is similar to the preceding case. The distinct real parts of the characteristic exponents are the growth rate intervals. The Morse sets in the finest Morse decomposition are the invariant subbundles corresponding to the different real parts.

EXAMPLE 2. We give an example where each $\lambda$ in the growth rate interval of a subbundle over $Q$ is the positive growth rate of a particular orbit. Let $Q$ be the annulus in $R^{2}$ defined, in polar coordinates, by $Q=\left\{(r, \theta) \mid r \in\left[r_{1}, r_{2}\right]\right.$ and $\theta \in[0,2 \pi]\}$. Let $E^{\prime}$ be a $R^{1}$-bundle over $Q$. Define $F$ on $E^{\prime}$ by $F_{t}((r, \theta), x)=\left((r, \theta+t), e^{r t} x\right)$ for $(r, \theta) \in Q$ and $x \in R^{1}$, where $\theta+t$ is taken modulo $2 \pi$. The growth rate interval for $E^{\prime}$ is $\left[r_{1}, r_{2}\right]$. For $(r, \theta) \in Q$, each orbit over the orbit $(r, \theta+t), t \in R$, has growth rate $r$. .

Example 3. Let $Q=S^{1}$ and let $f$ have two rest points on $S^{1}$ (Figure 3). Let $E^{\prime}$ be an $R^{1}$-bundle over $Q$. Define $F$ over $Q$ (Figure 4 ) by the

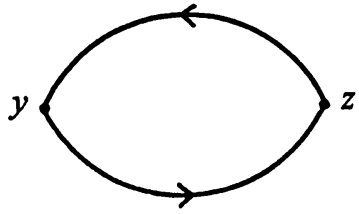

FIGURE 3
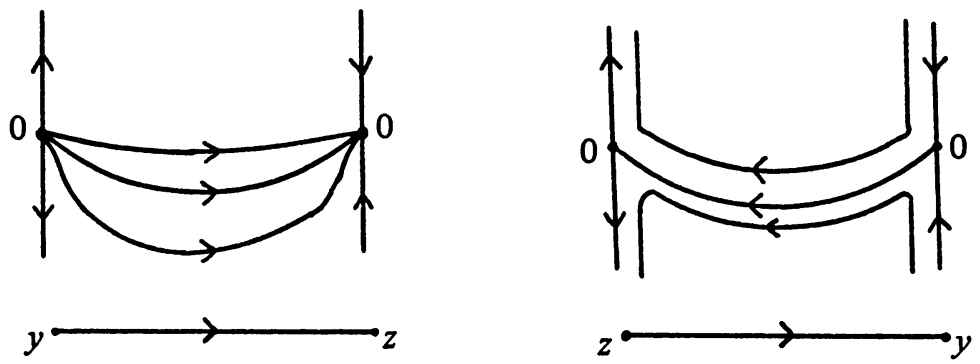

Figure 4 
solutions to the following differential equations: Over $y$, use $\dot{x}=x$; over $z$, use $\dot{x}=-x$; over the orbit from $y$ to $z$, use $\dot{x}=-(2 / \pi)(\arctan t) x$; and over the orbit from $z$ to $y$, use $\dot{x}=(2 / \pi)(\arctan t) x$. The growth rate interval for $E^{\prime}$ is $[-1,1]$. But the only positive growth rates for particular orbits are 1 and -1 .

12. An application to almost periodic differential equations.

DEFINITION. A set of real numbers is said to be relatively dense if there is an $L>0$ so that every open interval in $R$ of length $L$ contains at least one point of this set. ( $L$ is called a span of this set.)

Definition. A continuous function $h$ from $R$ into a metric space $(X, d)$ is almost periodic if for each $\epsilon>0$ there is an $L>0$ and a relatively dense set $\left\{\tau_{i}\right\}$ having span $L$ such that $d\left(h\left(t+\tau_{i}\right), h(t)\right)<\epsilon$ for all $t \in R$ and for all $\tau_{i}$.

Let $A$ be an almost periodic function from $R$ into the $n \times n$ matrices, considered as $R^{n^{2}}$. Consider the linear, nonautonomous differential equation $\dot{x}=A(\tau) x$ where $x \in R^{n}$ and $\tau \in R$. Let $C$ be the space of continuous functions from $R$ to $R^{n^{2}}$ with the topology of uniform convergence on compact subsets. Let $f$ be the Bebutov dynamical system on $C$, i.e., for $B \in C$, $f_{t}(B) \equiv B^{t}$ where $B^{t}(\tau)=B(t+\tau)$ for all $\tau \in R$. The Bebutov system is a continuous flow on $C$ [19]. If $A$ is almost periodic, the hull of $A$ (the closure of the orbit of $A$ ) is a compact subset of $C$ since $A$ is bounded and uniformly continuous. Since the orbit through $A$ is almost periodic as a function from $R$ into $C$, Birkhoff's recurrence theorem [16] implies that the hull of $A$ is a compact minimal set. Let $M$ denote the hull of $A$, a compact metric space. Let $E \equiv M \times R^{n}$. Define a linear flow $(F, f)$ on $E$ where $f$ is the Bebutov system restricted to $M$ and where $F$ is defined as follows: For $(B, x) \in M \times R^{n}, F_{t}(B, x)=\left(f_{t}(B), y(t)\right)$ where $y(t)$ is the unique solution to the differential equation $\dot{x}=B(\tau) x$ with initial value $\mathrm{\jmath}(0)=x . F$ is a continuous flow as shown in a more general situation in [19].

Since the zero section of $E$ being isolated is equivalent to each equation in the hull of $A$ having no nontrivial bounded solutions, the following is an immediate consequence of Theorem 10.2:

COROLlary 12.1. If no equation in the hull of $A$ has a nontrivial bounded solution, then the solution space of each equation in the hull has a hyperbolic splitting.

REMARK. More explicitly, Theorem 10.2 asserts that

(1) for each $B \in M, R^{n}=E^{s}(B) \oplus E^{u}(B)$, i.e., there is a splitting for $R^{n}$ varying continuously with $B$, and 
(2) there are positive real numbers $\alpha$ and $\lambda, \lambda<1$, so that each solution $y$ of $\dot{x}=B(\tau) x$ with initial value $y(0)$ in $E^{s}(B)$ satisfies $|y(t)| \leqslant$ $\alpha \lambda^{t}|y(0)|$ for all $t \geqslant 0$ and each solution $z$ with initial value in $E^{u}(B)$ satisfies $|z(t)| \geqslant \alpha^{-1} \lambda^{-t}|z(0)|$ for all $t \geqslant 0$.

By Theorem 9.7, $E^{s}$ and $E^{u}$ split into the direct sum of the invariant subbundles corresponding to the finest Morse decomposition of $P F$, and $P F$ is chain recurrent on each of these subbundles. In other words, for each equation in the hull, the stable and unstable solution spaces may split into subspaces with disjoint growth rate intervals, and the solutions in each subspace are chain recurrent when viewed as projective space (angular recurrence in dimension two). The growth rate intervals are the same for all equations in the hull, and we conjecture that the intervals are, in fact, points.

\section{REFERENCES}

1. M. Atiyah, K-theory, 2nd ed., Benjamin, New York, 1967. MR 36 \#7130.

2. R. C. Churchill, Isolated invariant sets in compact metric spaces, J. Differential Equations 12 (1972), 330-352.

3. C. C. Conley, The retrograde circular solutions of the restricted three-body problem via a submanifold convex to the flow, SIAM J. Appl. Math. 16 (1968), 620-625. MR 37 \#3113.

4. - On the continuation of invariant sets of a flow, Proc. Internat. Congress Math. (Nice, 1970), vol. 2, Gauthier-Villars, Paris, 1971, pp. 909-913.

5. - On a generalization of the Morse index, Ordinary Differential Equations, 1971, NRL-MRC Conference (L. Weiss Ed.), Academic Press, New York, 1972, pp. 27-33.

6. - The gradient structure of a flow. I, I. B. M. Research, RC 3932 (\#17806), Yorktown Heights, New York, July 17, 1972.

7. C. Conley and R. Easton, Isolated invariant sets and isolating blocks, Trans. Amer. Math. Soc. 158 (1971), 35-61. MR 43 \#5551.

8. J. Dugundji, Topology, Allyn and Bacon, Boston, Mass., 1966. MR 33 \#1824.

9. R. W. Easton, On the existence of invariant sets inside a submanifold convex to a flow, J. Differential Equations 7 (1970), 54-68. MR 40 \#2996.

10. - Locating invariant sets, Proc. Sympos. Pure Math., vol. 14, Amer. Math.

Soc., Providence, R. I., 1970, pp. 55-59. MR 42 \#5287.

11. - Regularization of vector fields by surgery, J. Differential Equations 10 (1971), 92-99. MR 47 \#4290.

12. S. Eilenberg and N. E. Steenrod, Foundations of algebraic topology, Princeton

Univ. Press, Princeton, N. J., 1952. MR 14, 398.

13. D. Husemoller, Fibre bundles, MicGraw-Hill, New York, 1966. MR 37 \#4821.

14. R. McGehee, Parabolic orbits in the three-body problem (to appear).

15. J. Montgomery, Cohomology of isolated invariant sets under perturbation, J. Differential Equations 13 (1973), 257-299.

16. V. V. Nemyckii and V. V. Stepanov, Qualitative theory of differential equations, OGIZ, Moscow, 1947; English transl., Princeton Math. Series, no. 22, Princeton Univ. Press, Princeton, N.J., 1960. MR 10, 612; 22 \#12258.

17. D. Rod, Pathology of invariant sets in the monkey saddle, J. Differential Equations 14 (1973), 129-170.

18. R. J. Sacker and G. R. Sell, Existence of dichotomies and invariant splittings for linear differential equations, J. Differential Equations 15 (1974), 429-458. 
19. G. R. Sell, Topological dynamics and ordinary differential equations, Van Nostrand Reinhold, London, 1971.

20. S. Smale, Differentiable dynamical systems, Bull. Amer. Math. Soc. 73 (1967), 747-817. MR 37 \#3598; erratum, 39, p. 1593.

21. J. A. Smoller and C. C. Conley, Viscosity matrices for two dimensional non-linear hyperbolic systems, Comm. Pure Appl. Math. 23 (1970), 867-884. MR 43 \#714.

22. - Viscosity matrices for two dimensional non-linear hyperbolic systems. II, Amer. J. Math. 44 (1972), 631-650.

23. - Shock waves as limits of progressive wave solutions of higher order equations, Comm. Pure Appl. Math. 25 (1972), 133-146.

24. E. H. Spanier, Algebraic topology, McGraw-Hill, New York, 1966. MR 35 \#1007.

25. T. Waźewski, Sur un principe topologique de l'examen de l'allure asymptotique des intégrales des équations différentielles ordinaires, Ann. Soc. Polon. Math. 20 (1947), 279-313. MR 10, 122.

26. F. W. Wilson, Jr. and J. A. Yorke, Lyapunov functions and isolating blocks, J. Differential Equations 13 (1973), 106-123.

\author{
DEPARTMENT OF MATHEMATICS, NORTH CAROLINA STATE UNIVERSITY, \\ RALEIGH, NORTH CAROLINA 27607
}

